\title{
An improved multiscale Eulerian-Lagrangian method for simulation of atomization process
}

\author{
Davide Zuzio $^{\mathrm{a}}$, Jean-Luc Estivalèzes ${ }^{\mathrm{a}}$, Bastien DiPierro ${ }^{\mathrm{b}}$ \\ ${ }^{a}$ Onera, The French Aerospatial Lab, Toulouse, Midi-Pyrénées, 31055, France \\ ${ }^{b}$ Claude Bernard University Lyon 1, Villeurbanne, Rhône-Alpes, 69100, France
}

\begin{abstract}
The physics of atomization process involve many spatial scales, generating a wide variety of liquid inclusions of different sizes with large density and viscosity ratios between liquid and gas phases. To correctly capture the dynamics of these phenomena, each scale should be resolved with an appropriate method to ensure the conservation of physical quantities (mass, momentum) as well as the jump conditions across the liquid-gas interface. To address these problems, an original multi-scale methodology has been developed. It consists of a core coupled Level-Set/Volume-of-Fluid method (CLSVOF) for accurate capture of primary atomization, an adaptive mesh refinement technique (oct-tree AMR) to dynamically optimize the structured Cartesian mesh and a particle tracking algorithm to capture droplet dynamics. An improved Eulerian-Lagrangian coupling has been developed to assure a smooth transition between the Eulerian and the Lagrangian modelling of the droplets, where both methods approach their design limits. The overall procedure is tested on simplified numerical tests and validated on a realistic planar liquid sheet atomization case. Results show its ability to reproduce the whole atomization process, from large scale instabilities to small droplet dynamics, and allow a preliminary statistical spray analysis.
\end{abstract}

Keywords: Atomization, Level-Set/VOF, Eulerian-Lagrangian coupling.

Email addresses: davide.zuzio@onera.fr, tel: +33562252845 (Davide Zuzio), jean-luc.estivalezes@onera.fr, tel:+33562252832 (Jean-Luc Estivalèzes), bastien.di-pierro@univ-lyon1.fr (Bastien DiPierro) 


\section{Introduction}

In the wide topic of multi-phase flows, atomization is a key process involved in many engineering applications, especially in combustion problems. The detailed numerical simulation of aeronautical combustion chambers has become a major topic in the last years, answering to the need to enhance the engine efficiency and to reduce the polluting agents. Among the most common injectors found in aeronautical engines are the airblast injectors. These devices are meant to use the assisted atomization to pulverize the fuel, which is obtained through the injection of a high pressurized liquid through a small fissure (either planar or annular) sandwiched by airflows. The role of the atomization process is to generate a cloud of fine droplets on a very small distance, in order to maximize the liquid surface and improve the combustion efficiency. The geometry of these injectors can be either planar or annular, both share similar developments of the atomization process.

The physics of atomization involves a wide range of phenomena and a strongly multi-scale dynamics, where many orders of magnitude span between larger and smaller structures (see Figure 1). Very different topologies such as drop, ligament, liquid sheet, may appear due to the many instabilities arising ([1], 2], [3], 4], 5]). The most common approach used in industrial oriented Large Eddy Simulations of reacting flows is to directly inject the spray with assumed characteristics, given by semi-empirical correlations. The liquid being modelled by a dispersed phase approach, no atomization is resolved within the simulation. Examples can be found in [6] and [7].

In more research-oriented simulations, focused on the atomization mech-

anisms, many numerical methods allow the Direct Numerical Simulation of these complex two phase flows, albeit in much more simplified and small scale configurations. The most widely employed methods are the Level-Set and the interface reconstructed Volume-of-Fluid methods, where the interface position and topology are tracked as accurately as possible in time. Among their most advanced evolutions are the Coupled Level-Set Method(8], the Conservative Level-Set [9] and the mass conserving Level-Set [10]. Thanks to those sharp-interface-capturing algorithms, numerical simulations of atomization have become possible: three-dimensional DNS simulations of liquid jet breakup have been reported in [11], [12], [10] and [13], while in [14] and [15] a full assisted atomization is simulated in different configurations. A way to efficiently perform such simulation of two phase flows is to use a variable spatial discretization which is adapted to the interface shape. This scenario 
has been investigated by [16] using a force refinement Level-Set grid method on structured and unstructured meshes, or by [17] with VOF method applied on an octree adaptative mesh refinement. Other works focus on the adaptive mesh refinement to maximize the resolution on the interface itself in order to lessen the computational burden ([18], [19], [20], [21], [22], [23]). However, these method have proved ineffective to to efficiently capture the formation and evolution of the droplet cloud, because they require a drastic mesh refinement of the large numerical domain, especially if the cloud of drop disperses uniformly.

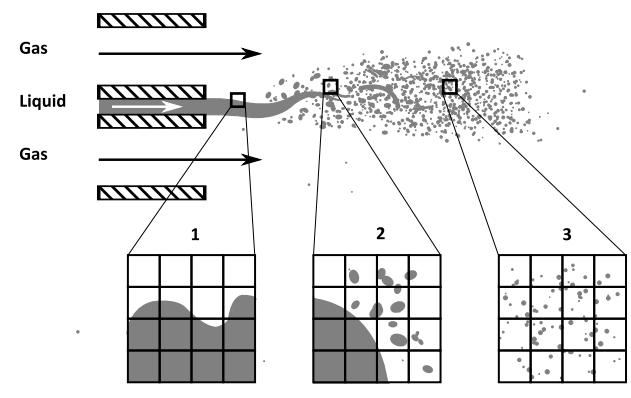

Figure 1: Representation of the multi-scale aspect of assisted atomization: interface characteristic shape versus a constant space scale.

An interesting and cheaper approach for the simulation of a dilute cloud of small, stable droplets is the dispersed phase. In this method the droplets are considered as small rigid spheres interacting with the gaseous carrying phase [24]. Different numerical implementations of this model exist, the most common being the Euler-Euler and Euler-Lagrange methods. Both can be seen as a discretization of the for the particle density function equation, 25] which describes the statistical properties of the spray in a volume of control in terms of particle position, size and velocity [26].

In the Euler-Lagrange approach the numerical implementation consists of an Eulerian resolution of the gaseous phase on an opportune mesh, while particles are treated in a Lagrangian framework. Each numerical particle (parcel) can represent an individual droplet or, in a more statistical approach, several physical particles [26]. The flow around the particles is not resolved, and physical modelling of the forces on the droplets and for the backward effect to the carrier flow is needed. When this latter is negligible, the carrying phase does not see the particles: it is referred as a one-way 
approach. Conversely, when the droplets effect is visible it is referred as a two-way approach. If the particle are supposed to be small, i.e. $d_{p} \ll L$ with $\mathrm{L}$ a characteristic length scale, the momentum change on an isolated particle is induced only by drag and gravitational forces [27]. At low particle Reynolds numbers, the drag force can be evaluated from the results of unsteady Stokes flow by means of the difference between the particle and the undisturbed carrying phase velocity. Conventionally, the droplet force is applied to the fluid as a point source located at the center of mass of the droplet [28].

In the discrete formulation, the particles are usually small compared to the cell size, the approach relying on sub-grid models for the small scale particle-fluid interaction. The individual particle effect on the gas is then small, and the cell velocity is a good approximation of the undisturbed velocity. The flow variations due to the presence of the particle are also contained within the cell. While this is certainly the case in situations like a large combustion chamber being resolved with a cost-effective grid, when dealing with atomization a resolution on the scale of a few droplet diameters is required. In this situation, the point-source approximation becomes increasingly deficient, since the ratio of the droplet volume to the gas-cell volume increases [28]. Indeed, this situation imposes a limitation on the use of the point-source approximation. The error of the approximation occurs in two ways; on one hand, the gas cell becomes strongly (or entirely) affected by the particle velocity; on the other hand, the particle have a domain of influence on the flow larger than the cell itself.

Within DNSs of atomization, as the detailed simulation of all the spatial scales remains very expensive, the particle tracking method appears interesting for the smallest particles. Indeed, these structures are sufficiently small by regard with the smallest computational mesh size and have a spherical shape due to surface tension effects, so that the dispersed phase model assumptions are validated. Following this idea, the problem can be numerically split in two scales, following the two stages of atomization, in order to adapt the local two-phase modelling. A separated phases model as the VOF or LevelSet could describe the primary atomization, where the two phases are clearly distinguished and the interface subject to low frequency instabilities, while the dispersed phase model would be better adapted to describe the evolution of the spray in terms of secondary atomization, transport and eventually evaporation. In [14] (by extension of [16] with a Level-Set method), [29] and 30] the coupled DNS and Lagrangian method technique has been success- 
fully applied to the atomization of jets in cross-flow configuration. In [15] (coupled to a VOF method [17] ) and in [31] a similar approach involving VOF and CLSVOF methods were developed to resolve assisted atomization. Both of them tracked point particle liquid particles with a drag force to couple Eulerian and Lagrangian quantities, this methodology seeming accurate enough to capture both large and small inclusion. In other approaches, the generation of the spray is not direct but it is instead treated by a more statistical approach, as in [32] and 33], in order to avoid the use of cell sizes small enough to resolve the smallest drops. However, in most of those approaches the transition from Eulerian to Lagrangian formulation is still controversial as well as the treatment of inclusions exceeding the grid size.

Indeed, if one consider a medium liquid drop, i.e. a quasi-spherical inclusion whose radius is equivalent to a few mesh cell, the Eulerian formulation is not able to correctly predict the dynamics. Actually, at least $6-8$ mesh points per radius are necessary to described the evolution of such drop to avoid disappearance of liquid inclusion due numerical diffusion of Level-Set method, or less accurate interface representation of the piecewise linear reconstruction of VOF method. On the other hand, the point-particle assumption of the dispersed phase may induce the errors previously described.

Hence, a third modelling scale have to be introduced so that the spatial gap between the two models is filled, as it seems inappropriate. The present work proposes an approach to model this third scale between pure Eulerian and Lagrangian representations: medium drops are supposed to have a rigid spherical shape, and they are followed by rigid translation while their mass and momentum properties are projected on the Eulerian field. The Navier-Stokes solution of the flow is then used to evaluate the forces acting on the droplet and thus their velocity. This formulation results in a more conservative representation of the initial characteristics of the droplet. The hypothesis of spherical shape allows an efficient coupling with an AMR technique: a stable Eulerian droplet is transformed to Lagrangian droplet on the finest level of refinement. Then, it is advected by the medium droplet approach, which allows a fully two-way resolution of the two phase field. When the droplet is far enough from the resolved liquid core, the mesh coarsening is progressively triggered. When the local grid size becomes larger than the droplet, the classical dispersed phase point-particle method takes over. The inverse transformation has been adopted to treat the collision between Lagrangian particle with the interface of a large liquid inclusion, where the medium drop algorithm takes over if the mesh size becomes fine enough. 
The interest of this approach is twofold. On one side, the DNS simulations of atomization can be used to generate a dispersed phase ready to be analysed, as position and velocity of the particles are easily stored and available at any time. This data could be ideally used as initial condition for a LES type simulation. On the other side, the analysis of the numerical droplets can furnish complete data-sets to be used to build large-scale atomization models, in a region where experimental devices are usually not able to perform measurements (too dense sprays). For this reason it is important to have initial conditions of the droplets as much accurate as possible.

In this paper, a CLSVOF method is used (from [34] and [8]) to accurately predict the interface shape with the Level-Set description and to ensure local mass conservation with the VOF formulation. An adaptive mesh refinement (given by the PARAMESH package [35]) is dynamically performed around the resolved CLSVOF interface throughout the computation, so that jump conditions are accurately satisfied. The smallest particles are tracked with a Lagrangian formulation and a classical drag force, while medium ones are treated using the mixed Eulerian-Lagrangian method previously described. The whole procedure has been parallelized to allow relatively large computational domains. The numerical code is an evolution of the one described in [23] and 36].

This paper is organized as follows: section 2 details the governing equation of two-phase flow, section 3 describes the numerical procedure for large scale interfacial approach (CLSVOF method, pressure solver, adaptative mesh refinement). Section 4 details the original conservative treatment for the medium inclusions as well as the more classical Lagrangian particle tracking

for the small drops. In this section, the Euler to Lagrange transition criteria for the inclusions (size, shape) are presented; the recombination of a particle with a large scale interface is described as well. Finally, section 5 shows some academic two-phase problems and show the capability of the present method to described multi-scale problems, in particular the assisted atomization of a planar liquid sheet.

\section{Governing equations}

The numerical methodology proposed here aims to solve two-phase flows under the incompressibility hypothesis. The Navier-Stokes equations in each 
phase under free divergence velocity field constraint are considered:

$$
\begin{aligned}
\frac{\partial \mathbf{u}}{\partial t}+(\mathbf{u} \cdot \nabla) \mathbf{u}-\frac{1}{\rho} \nabla \cdot \mathbf{T} & =\mathbf{f}, \\
\nabla \cdot \mathbf{u} & =0,
\end{aligned}
$$

where $\boldsymbol{u}=\left[u_{i}, u_{j}, u_{k}\right]^{T}$ represents the velocity vector field, $p$ the hydrodynamic pressure, $\rho$ the fluid density, $\mu$ the fluid dynamic viscosity and $\mathbf{f}=\left[f_{i}, f_{j}, f_{k}\right]^{T}$ the external body forces like gravity.

$$
\begin{aligned}
& \mathbf{T}=-p \mathbf{I}+\mathbf{D} \\
& \mathbf{D}=\mu\left(\nabla \mathbf{u}+(\nabla \mathbf{u})^{T}\right)
\end{aligned}
$$

Those equations are solved in both liquid and gases phases. Within this paper, the liquid and gazes properties will be subscribed with "l" and "g" respectively. Across the interface, the no slip condition imposes the continuity of velocity field $\mathbf{u}_{l}=\mathbf{u}_{g}$, while jump conditions are imposed by viscous constraints equilibrium and surface tension effects ( $\sigma$ being the liquid-gas surface tension coefficient):

$$
\begin{aligned}
{[p]-\mathbf{n} \cdot[\mu(\nabla \mathbf{u}+(\nabla \mathbf{u}))] \cdot \mathbf{n}=\sigma \kappa, } \\
\mathbf{t} \cdot[\mu(\nabla \mathbf{u}+(\nabla \mathbf{u}))] \cdot \mathbf{n}=0,
\end{aligned}
$$

where $\kappa$ is the interface curvature, $\mathbf{t}$ and $\mathbf{n}$ the tangential and normal unit vectors oriented from gas to liquid and [.] $=(.)_{l}-(.)_{g}$ any jump across the interface.

\section{Eulerian modelling}

\subsection{Interface tracking}

An interface tracking method is employed to solve the primary atomization region, up to the formation of the droplets. The coupled LevelSet/Volume-Of-Fluid (CLSVOF) formulation is used in reason of its accurate description of the interface shape, given by the Level-Set function, and the crucial mass conservation properties given by the VOF. The chosen implementation of the CLSVOF follows [8] and the improved version from [34], with some further modifications described in [36]. The Level-Set function 
$\phi(\mathbf{x}, t)$ gives implicitly the interface position, as it is defined as a signed distance to the interface. The evolution of $\phi$ follows a linear advection equation driven by the divergence free fluid velocity:

$$
\frac{\partial \phi}{\partial t}+\mathbf{u} \cdot \nabla \phi=0
$$

The physical properties of each fluid are determined upon the sign of $\phi$ :

$$
\alpha(\mathbf{x}, t)= \begin{cases}\alpha_{l} & \text { if } \quad \phi>0 \\ \alpha_{g} & \text { elsewhere }\end{cases}
$$

The regularity of the Level-Set function allows a straightforward computation of the geometrical properties at the interface, namely normal vector and curvature:

$$
\mathbf{n}=\frac{\nabla \phi}{\|\nabla \phi\|}, \quad \kappa=\nabla \cdot\left(\frac{\nabla \phi}{\|\nabla \phi\|}\right)
$$

The VOF function $C$ represents the ratio of liquid volume inside a computational cell over the total cell volume

$$
C_{i}=C\left(\mathbf{x}_{i}\right)=\frac{V_{l, i}}{V_{i}}
$$

where $\mathbf{x}_{i}$ is a cell centered and $V_{i}=V_{l, i}+V_{g, i}$. This function is driven by the same linear passive advection equation as the Level-Set (5):

$$
\frac{\partial C}{\partial t}+\mathbf{u} \cdot \nabla C=0
$$

\subsection{Temporal integration}

Let $\left(\mathbf{u}^{n}, p^{n}, \phi^{n}, C^{n}\right)$ be the solution at a given time $t^{n}, n$ being the temporal subscript. The necessary steps to integrate the flow equations can be regrouped into two steps. At fist, the interface motion given by the two equations (5) and (9) is solved; subsequently, the incompressible flow equation (1) is solved using the updated interface position. The CLSVOF steps can be summarized as:

1) The Level-Set function is used to evaluate the surface representing the PLIC reconstructed interface by solving a least-square problem on the local nine-point stencil (as described in [8]; a local stencil correction has been introduced to better resolve some difficult configurations, such as when two interfaces cross the base stencil). 
2) Semi-Lagrangian fluxes are computed at each cell face by geometric computations [37].

3) The Level-Set and VOF functions are integrated simultaneously using the previously computed fluxes: equations (5) and (9) are solved alternatively in each space direction with a second order conservative split scheme;

4) The updated VOF value is used to offset the local value of the Level-Set ("enforcing"), in order to assure local mass conservation.

Once $C^{n+1}$ and $\phi^{n+1}$ are obtained, they are used to obtain $\rho^{n+1}$ and $\mu^{n+1}$ through the ghost fluid method as in [23]. Then, the flow equations are solved by a Chorin projection method:

1) Equation (11) is computed without pressure term, using a fully explicit forward Euler scheme, in order to predict the velocity field $\mathbf{u}^{*}$ :

$$
\begin{aligned}
\mathbf{u}^{*} & =\Delta t \mathbf{F}^{n} \\
\mathbf{F}^{n} & =-\left(\mathbf{u}^{n} \cdot \nabla\right) \mathbf{u}^{n}+\frac{1}{\rho^{n+1}} \nabla \cdot\left(\mu^{n+1}\left(\nabla \mathbf{u}^{n}+\left(\nabla \mathbf{u}^{n}\right)\right)\right)+\mathbf{f}^{n},
\end{aligned}
$$

The viscous term is evaluated using a spatial second order centered scheme and the convective one with a 5th order WENO scheme (see again 23] for the explicit discretization).

2) The pressure is evaluated by solving the elliptic equation

$$
\nabla \cdot\left(\frac{1}{\rho^{n+1}} \nabla p^{n+1}\right)=-\frac{\nabla \cdot \mathbf{u}^{*}}{\Delta t}
$$

This equation is solved using a Krylov BiCG-Stab preconditionned by a multilevel algorithm built on the AMR tree. The interested reader is referred to [23] for more details.

3) Finally, the divergence-free velocity field is obtained by correcting the predicted one :

$$
\mathbf{u}^{n+1}=\mathbf{u}^{*}-\Delta t \frac{1}{\rho^{n+1}} \nabla p^{n+1}
$$


4) Steps 1-3 are repeated twice in a fully coupled second-order RungeKutta predictor-corrector scheme, each sub-step re-evaluating the convective velocity used to advect the CLSVOF $\left(t=t^{n}\right)$ and giving the final velocity:

$$
\begin{aligned}
& \mathbf{u}^{n+\frac{1}{2}}=\mathbf{u}^{n}+d t \mathbf{F}^{n} \\
& \mathbf{u}^{n+1}=\frac{1}{2} \mathbf{u}^{n+\frac{1}{2}}+\frac{1}{2} d t \mathbf{F}^{n+\frac{1}{2}}
\end{aligned}
$$

\subsection{Adaptive Mesh Refinement}

The dynamic adaptation of the mesh resolution (AMR) is performed through the open source parallel PARAMESH package. The whole numerical procedure is detailed in [23].

The computational domain is recursively decomposed in an arbitrary number of blocks, resulting into an oct-tree data structure. Each block is a Cartesian domain with a user-defined fixed number of cell in the three directions but with a variable grid spacing (Figure 2). The maximal and minimal grid spacing is fixed at the beginning of the simulation. In case of need, during checkpoints they can be altered with a small effort. The refinement ratio is fixed at 2, so that any neighbour of a given cell can't be more than two times larger or smaller than its size. The refinement process is automatically performed following one or more user-defined criteria. Within this work, the refinement is forced to be at the maximum level around the interface, where the discontinuities are located, while the mesh is allowed to de-refine into the single phase regions. The block numbering and parallel repartition is performed using a Morton type algorithm and the data transfer from a coarser grid to a finest one (or reciprocal) is done through interpolation process: the velocity field is subject to second-order divergence-free interpolation from [38, the scalar fields to conservative second-order Q1 interpolation. Any

interested reader is referred to the PARAMESH documentation or [35] and 39] for more details.

\section{Multi-scale approach}

As mentioned in section 3.3 , the refinement process is automatically triggered around the interface in order to correctly resolve the small liquid scales. However, in realistic long-time simulations the atomization process is expected to generate droplets of dimension comparable to some mesh cell sizes $(L \sim \Delta x)$, which can not be correctly resolved by the CLSVOF (or any 

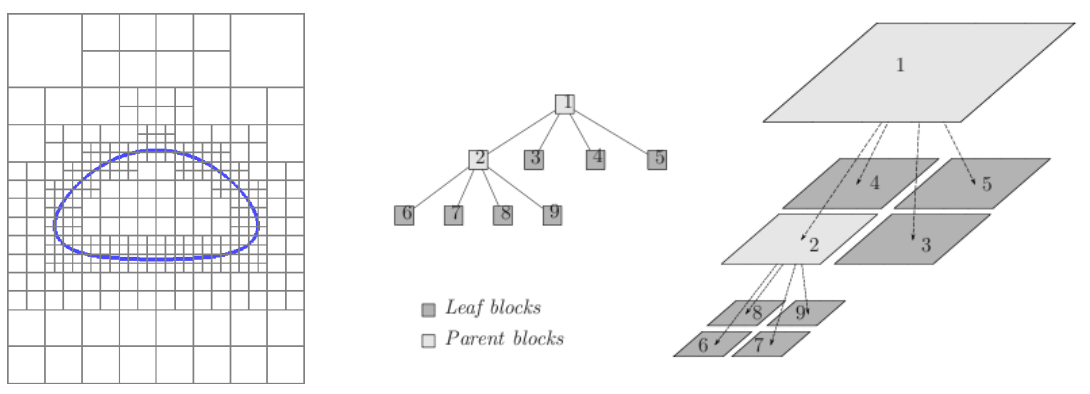

Figure 2: Left: Illustration of the refinement around the interface on a two dimensional rising bubble test case. Right: example of an octree block structure with Morton numbering

other Eulerian interface-tracking method) because of the insufficient resolution. Worse, those small structures force a useless local grid refinement so that, in numbers, they may defeat the purpose of the AMR by refining almost the whole computational domain. A second point is that the interface tracking method can not track the formation of very small liquid inclusions of size inferior to the finest mesh cell, which are formed when liquid structures like membranes or ligaments are locally under resolved.

A relevant solution to both problems is to couple the mesh refinement strategy with a dispersed phase approach for the mall liquid inclusions. In this approach, a small droplet is assumed to be a stable rigid sphere, in the sense that the gas shearing force is considered to be no more strong enough to overcome the surface tension and thus induce a secondary atomization. The characteristics of the droplet are then given by its center of mass, its radius and velocity. Resolving the dispersed phase by a Lagrangian modelling, the droplet motion can be tracked by advecting its center of mass with a drag-force model on a very coarse mesh, for a fraction of the Eulerian computational cost. This approach allows the resolution of the primary atomization up to the droplet formation by the CLSVOF and the most refined mesh, while the droplets can be tracked far outside the primary atomization region by the Lagrangian algorithm on a cheaper de-refined mesh. Figure 3 shows the principles of this approach.

The most basic transition involves the removal of the liquid phase of the droplet, resolved by the CLSVOF on a small number of cells $(L \approx \Delta x)$, from the Eulerian field and the injection of a volume-less point particle in the position corresponding to the center of mass of the structure. The mesh adap- 

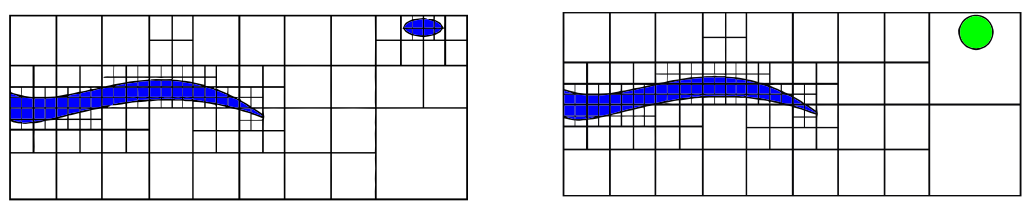

Figure 3: Left: Illustration of the refinement around a (under-)resolved droplet. Right: Illustration of the coupled effect of AMR and point-particle Lagrangian tracking

tation is supposed in the next time-steps to progressively de-refine the mesh until the particle physical radius become smaller than the mesh size, and the assumption of dispersed phase holds. However, some important issues arises in the transition between the Eulerian and the Lagrangian formulation if care is not taken:

1) The direct transformation on the refined grid artificially changes the local flow topology, as the domaine of droplet influence being reduced from the initial Eulerian volume to the cell containing the center of mass only. Moreover, the source term becomes very stiff.

2) The drag-force model requires an undisturbed velocity to compute the correct dynamics of the droplet. This velocity is not directly available from the cell containing the point particle, nor the surrounding cells. The resulting droplet acceleration is in consequence incorrect.

3) If a larger resolved liquid structure is present near the droplet, the mesh coarsening is not allowed until the droplet or the structure leaves the block. The radius to cell size ratio remains in this case unchanged.

If the first problem can be addressed by integrating the source terms over a set volume of influence [40], [26], the second is trickier. One possible solution is given in [41], where the actual influence of the droplet on the underlying Eulerian field is explicitly subtracted in order to obtain the undisturbed velocity by its own definition. For the droplet whose size is lesser than the mesh cell, the Lagrangian approximation can be at first considered as correct, as only the cell velocity is affected.

The approach proposed in this paper proposes an intermediate treatment between the pure Eulerian and the point-particle drag force models. This approach is used whenever a droplet is detected by the CLSVOF on the finest AMR mesh level, and the transformation criteria (described further in 
the paper) are met: at this stage, the droplet normally covers multiple cells. If the droplet exits the refined AMR zone, when its size becomes smaller than the mesh size the algorithm reverts to a point-particle drag-force model. For the sake of clarity, we define the Lagrangian droplets as:

1) "small inclusion": the droplet size is lesser than the local mesh cell size (drag-force model);

2) "medium inclusion": the droplet size is greater than the local mesh size (intermediate treatment).

In the first case, the dispersed phase approach hypothesis are considered as satisfied; in the second, a kind of Euler-Lagrange penalty method has been developed to allow the more complex interaction of the medium droplets and the underlying flow. The approach is represented in Figure 4. In Figure 4(a)

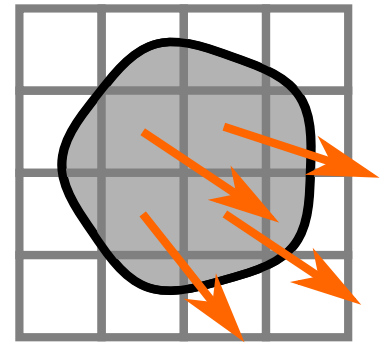

(a)

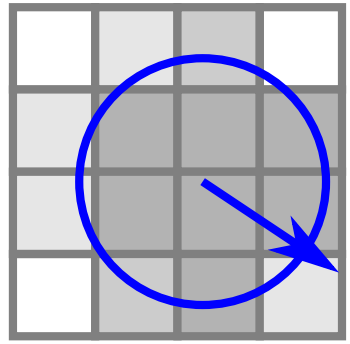

(b)

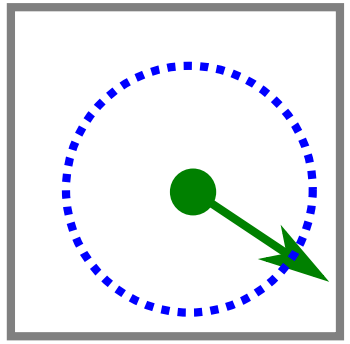

(c)

Figure 4: Representation of the three steps of the mulit-scale methodology: (a) a CLSFOV drop is formed on the fine mesh; (b) it is transformed into a Lagrangian medium drop (on the same fine mesh) with its own domain of influence on the Eulerian fields; (c) the drop travels to a coarsened zone: when it becomes smaller than the local mesh cell, a standard point-particle algorithm is employed (the particle volume is neglected).

a liquid inclusion spanning several cells is detected into the Eulerian field on a fine AMR mesh. If the detection criteria are satisfied, the inclusion is transformed into a "medium" droplet, defined by a center of mass, a radius and an unique interpolated velocity. The cells obscured by its volume retains the characteristics of liquid cells in the momentum equation (1), meaning that the flow still see a liquid inclusion of the actual size of the droplet, as it can be seen in figure Figure 4(b). The velocity of the droplet at each time-step is the result of interpolation of the underlying velocity field. When a medium droplet travels to a coarsened region or the inclusion is smaller than the finest 
grid size, it became a volume-less point particle driven by a drag force model, Figure $4(\mathrm{c})$.

The proposed multi-scale approach includes the Lagrangian to Eulerian inverse conversion. This is needed because in the primary atomization process the droplets co-exist with larger structures: this may lead to coalescence processes, in which the particles collide a resolved liquid body. The collision is handled differently for the small and medium particles. In the former situation, the droplet is deleted, and its relatively small mass and momentum are added to the local cell; in the latter, the collision is naturally resolved by the Eulerian solver by projecting the droplet back to the Eulerian field on the fine mesh by reconstructing the local Level-Set and VOF. In the following paragraphs all the numerical algorithms are detailed.

\subsection{Detection of droplets}

The first step of the multi-scale algorithm is to detect the liquid inclusion to be transformed. A tag-propagation algorithm is employed. As illustrated on Figure 5, each liquid inclusion is detected by propagation of a tag over each liquid-cell, i.e. a cell where $C_{i}>0.5$. At first, each liquid-cell is tagged with an unique number. Then, the smallest tag is iteratively propagated among each neighbouring liquid-cells. When the tag is entirely propagated among all liquid cells which constitute the liquid inclusion, the tag is then extended to all surrounding cells where $0<C_{i}<0.5$. Considering a liquid inclusion $l$, one can evaluate the volume of this inclusion $\nu_{l}$ and its center of mass $\mathbf{x}_{l}$ over all cells $i_{l}$ which contains the liquid inclusion through the VOF function:

$$
\begin{aligned}
\nu_{l} & =V_{\text {cell }} \sum_{i \in i_{l}} C_{i} \\
\mathbf{x}_{l} & =V_{\text {cell }} \frac{1}{\nu_{l}} \sum_{i \in i_{l}} \mathbf{x}_{i} C_{i}
\end{aligned}
$$

where $V_{\text {cell }}$ is the volume of a mesh-cell. Most of the droplets are supposed to be still resolved on multiple cells when detected $\left(\nu_{l}>V_{\text {cell }}\right)$ : these are the "medium" inclusions. If any floatsam is produced by the CLSVOF algorithm, this can be detected as well: an isolated $0<C_{i}<0.5$ cell not attached to any larger structure, i.e. surrounded by cells with $0<C_{i}<0.5$. In this case the Level-Set function does not see any liquid mass, so that for the CLSVOF this liquid would be effectively lost. We track these small particles anyway. 

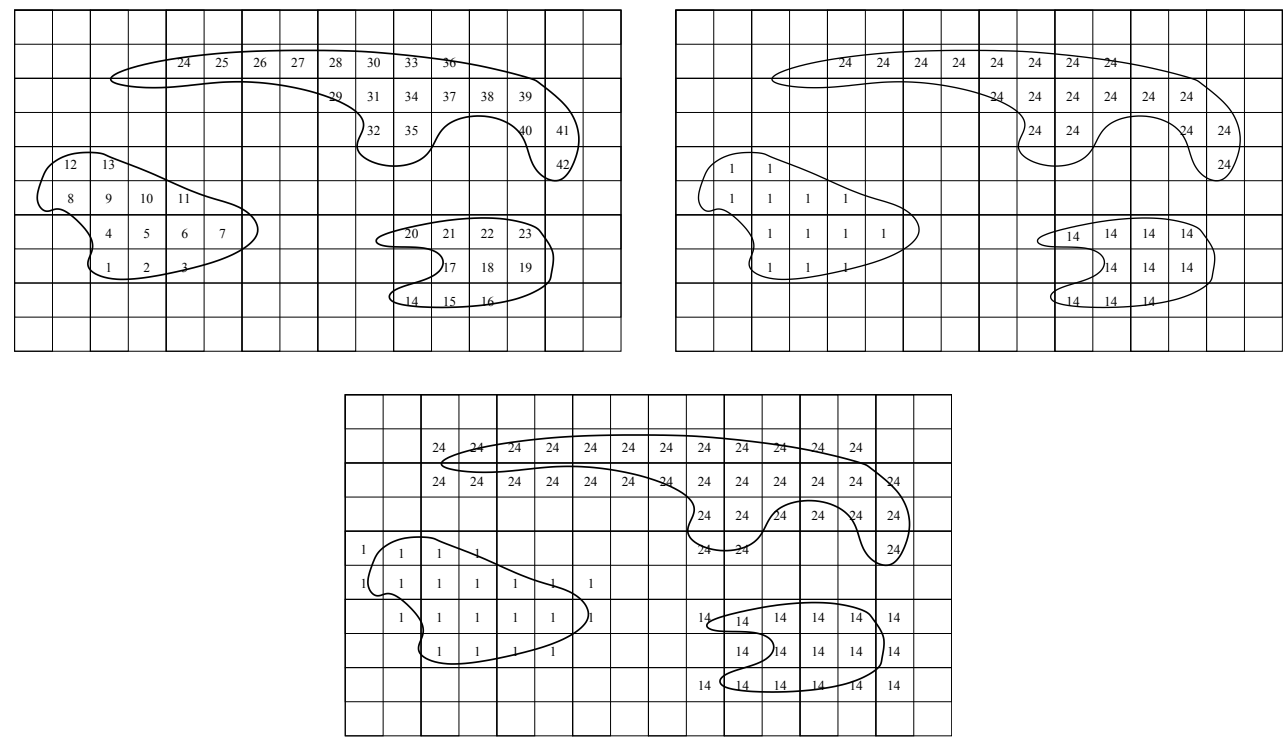

Figure 5: Illustration of liquid inclusion detection. Each "liquid cell" $\left(C_{i}>0.5\right)$ is tagged with a unique number (top left). The smallest tag is propagated among each neighboring liquid cell (top right). Finally, this tag is propagated to each cell where $0<C_{i}<0.5$ (bottom).

\subsection{Transformation criteria}

In a typical atomization process, the flow is highly disturbed by many instabilities (Kelvin-Helmholtz, Rayleigh-Taylor, Plateau-Rayleigh ...). Hence, it is quite difficult to determine a physical parameter (like Weber number) to predict the stability of any drop and allow the transition between Eulerian and Lagrangian resolution. Hence, a possible transition is determined here on geometrical considerations. Let $N_{l}$ be the total number of cell contained in a liquid inclusion $l$. For very small inclusions $N_{l} \leq 1$ the inclusion is automatically treated in a Lagrangian point particle way, without any other arguments. For medium inclusion $1 \leq N_{l} \leq 8^{31}$, a sphericity criterion is used, as illustrated on Figure 6. To this way, a sphere of equivalent volume is superimposed on the center of mass of the liquid inclusion, for which the radius is

$$
r_{p}=\left(\frac{3}{4} \frac{\nu_{l}}{\pi}\right)^{1 / 3}
$$

\footnotetext{
${ }^{1}$ This value is currently limited by
} 

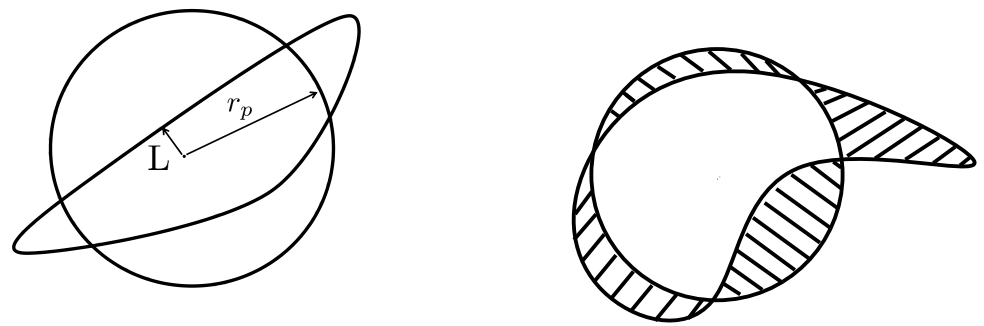

Figure 6: Sphericity criterion for medium inclusion. A sphere of equivalent volume is superimposed on the considered inclusion. The inclusion is becomes a Lagrangian particle if the difference in length $r_{p}-L$ and volume (dashed zone) are sufficiently small

and the difference of characteristic length $\delta_{l}$ and volume $\delta_{V}$ (dashed part on the right of Figure 6 are computed :

$$
\left\{\begin{array}{l}
\delta_{l}=r_{p}-L \\
\delta_{V}=V_{\text {cell }} \sum_{i \in i_{l}}\left|C_{i}-\chi_{i}\right|
\end{array}\right.
$$

where $\chi_{i}=1$ if $\left\|\mathbf{x}_{i}-\mathbf{x}_{l}\right\| \leq r_{p}, 0$ otherwise and $L$ is the smallest length between the center of mass and the interface, i.e. $L=\phi\left(\mathbf{x}_{l}\right)$. The inclusion is assumed as quasi-spherical if

$$
\frac{\delta_{l}}{r_{p}}<\alpha \frac{\delta_{V}}{\nu_{l}}<\beta .
$$

After some numerical test, $\alpha$ and $\beta$ have been set between 0.5 and 1 (increasing these values force more conversions). If those two criteria are satisfied, the inclusion becomes a Lagrangian particle of volume $\nu_{l}$, radius $r_{p}$ and initial velocity $\mathbf{v}_{p}$ (the definition of $\mathbf{v}_{p}$ is given in the following paragraphs) at the location $\mathbf{x}_{p}=\mathbf{x}_{l}$. If they are not satisfied, the inclusion is still treated by the Eulerian CLSVOF resolution until a possible stabilization or fragmentation occur.

\subsection{Tracking}

The advection of any droplet, whether medium or small, follows the equation

$$
\frac{d \mathbf{x}_{p}}{d t}=\mathbf{v}_{p}
$$

All the droplets are supposed to be spherical and undergo rigid translation by advection of their center of mass at each time-step. However, the definition of the advecting velocity $\mathbf{v}_{p}$ is different for the two class of Lagrangian particles. 


\subsubsection{Medium droplets}

An original tracking method has been developed for the medium drop, in a way of a penalty method. Indeed, the idea of this method is to project the liquid properties on the Eulerian field within the droplet actual diameter. Hence, for a given particle $(\cdot)_{p}$ of center $x_{p}$ and radius $r_{p}$, the cells within its domain of influence can be detected:

$$
\mathcal{D}_{p}=\left\{\forall i \mid\left\|\mathbf{x}_{i}-\mathbf{x}_{p}\right\|<\left(r_{p}+\sqrt{3} / 2 d x\right)\right\} 2
$$

The distance to the center evaluated in all the influenced cells corresponds

to a local particle Level-Set function, which can be used to precisely define local quantities:

$$
\hat{\phi}_{p, i}=\left\|\mathbf{x}_{i}-\mathbf{x}_{p}\right\|
$$

Any quantity $(\cdot)_{i}$ at the discrete point $i$ can be evaluated by weighted averages as:

$$
(\cdot)_{i}=(\cdot)_{l} H\left(\hat{\phi}_{p, i}\right)+(\cdot)_{g}\left[1-H\left(\hat{\phi}_{p, i}\right)\right]
$$

with $H(\mathbf{x})$ a smoothed Heavyside function. The face-centered densities and viscosities $\rho_{i+1 / 2}, \mu_{i+1 / 2}$ can be evaluated as:

$$
\begin{aligned}
\rho_{i+1 / 2} & =\frac{\rho_{i}\left|\hat{\phi}_{p, i}\right|+\rho_{i+1}\left|\hat{\phi}_{p, i+1}\right|}{\left|\hat{\phi}_{p, i}\right|+\left|\hat{\phi}_{p, i+1}\right|} \\
\mu_{i+1 / 2} & =\frac{\mu_{i}\left|\hat{\phi}_{p, i}\right|+\mu_{i+1}\left|\hat{\phi}_{p, i+1}\right|}{\left|\hat{\phi}_{p, i}\right|+\left|\hat{\phi}_{p, i+1}\right|}
\end{aligned}
$$

For cell-centered quantities, a more conservative weighting can be obtained by directly using the local volume of fluid:

$$
\begin{aligned}
& \rho_{i}^{n+1}=\rho_{l} C_{i}+\rho_{g}\left(1-C_{i}\right) \\
& \mu_{i}^{n+1}=\mu_{l} C_{i}+\mu_{g}\left(1-C_{i}\right)
\end{aligned}
$$

Equations (11) and $(12)$ are subsequently solved on the Eulerian mesh with the new values of density, viscosity and velocity updated with respect to particle positions. The final pressure inside the drops is corrected by Laplace relation:

$$
p_{i}^{n+1} \rightarrow p_{i}^{n+1}+2 \frac{\sigma}{r_{p}}, \quad \forall i \in \mathcal{D}_{p}
$$

\footnotetext{
${ }^{2}$ The $\sqrt{3} / 2 d x$ term allows to take in account partially filled cells $C_{i}<0.5$.
} 
Afterwards, equation 13 is solved with the corrected pressure. Then, the new particle velocity is obtained by averaging the Eulerian velocity inside the considered drop using equation 22 weighting:

$$
\mathbf{v}_{p}=\frac{\sum_{i} H\left(\hat{\phi}_{l}\right) \mathbf{u}_{i}^{n+1}}{\sum_{i} H\left(\hat{\phi}_{l}\right)}, \quad \forall i \in \mathcal{D}_{p}
$$

Finally, equation 20 is solved with this new particle velocity.

\subsubsection{Small droplets}

For the small droplets, i.e. $r_{p} \leq \Delta_{x, \text { min }}$, a classical drag coefficient for spherical point particle is used, from [42]:

$$
C_{D}\left(R_{e, p}\right)= \begin{cases}24 R_{e, p}^{-1}\left(1+0.15 R_{e, p}^{0.687}\right) & \text { if } \quad R_{e, p}<1000 \\ 0.445 & \text { otherwise }\end{cases}
$$

with

$$
R_{e, p}=\frac{2 r_{p} \rho_{g}\left\|\mathbf{v}_{p}-\mathbf{v}_{p, e}\right\|}{\mu_{g}}
$$

and where $v_{p, e}$ the Eulerian velocity interpolated at the particle location. These values give a relaxation time in the form

$$
\tau_{p}=\frac{4 \rho_{l}}{3 \rho_{g}} \frac{2 r_{p}}{C_{D}\left\|\mathbf{v}_{p}-\mathbf{v}_{p, e}\right\|}
$$

Then, the updated particle velocity can be obtained by

$$
\frac{d \mathbf{v}_{p}}{d t}=\tau_{p}^{-1}\left\|\mathbf{v}_{p}-\mathbf{v}_{p, e}\right\|
$$

The updates of particle $p$ velocity and realized by the following:

$$
\begin{aligned}
& \mathbf{v}_{p}^{n+1}=\mathbf{v}_{p}^{n}+d t\left[\left\|\mathbf{v}_{p}-\mathbf{v}_{p, e}\right\| \tau_{p}^{-1}+g\left(\frac{\rho_{p}-\rho_{g}}{\rho_{l}}\right)\right] \\
& \mathbf{x}_{p}^{n+1}=\mathbf{x}_{p}^{n}+d t \mathbf{v}_{p}^{n+1}
\end{aligned}
$$

Equations (34) and (35) are integrated using a classical second order RungeKutta scheme with a mid-course velocity update. The two-way disturbance of any small particle $p_{i}$ located into a cell $x_{i}$ on the carrying flow consists of an added term on the velocity equation (1):

$$
\mathbf{u}_{i}=\mathbf{u}_{i}+d t \frac{\left\|\mathbf{v}_{p}-\mathbf{u}_{i}\right\|}{\tau_{p}} \frac{V_{p} \rho_{l}}{V_{i} \rho_{i}}
$$

$V_{p}$ and $V_{i}$ being respectively the volume of the particle and of the cell and $\rho_{i}$ from equation 26 the average cell density. 


\subsection{Drop-interface collision}

\subsubsection{Medium droplets}

In the present work, a re-impact algorithm is implemented to take into account of a possible collision between particles and the liquid-gas interface. The re-impact consists of the inverse transformation in which a droplet is removed from the Lagrangian framework and re-created in the CLSVOF formalism. The re-impact is activated for any particle crossing the interface or being sufficiently close, while moving on a collision course, as illustrated of Figure 7

$$
\begin{aligned}
\mathbf{v}_{p} \cdot \nabla \phi & >0 \\
\left|\phi_{p}\right| & <r_{p}+\Delta_{x, \text { min }}
\end{aligned}
$$

where $\phi_{p}$ is the interpolation of $\phi$ at a location $\mathbf{x}=\mathbf{x}_{p}$ and $\Delta_{x, \text { min }}$ the grid size. If those criterion are satisfied, both Level-Set and VOF functions are reconstruct as follows:

$$
\begin{aligned}
\phi_{i}^{n} & =r_{p}-\left\|\mathbf{x}_{p}-\mathbf{x}_{i}\right\| \\
C_{i}^{n} & =\mathcal{H}\left(\phi_{i}^{n}\right)
\end{aligned}
$$

for all cells $i$ inside the drop (i.e. || $\mathbf{x}_{p}-\mathbf{x}_{i} \|<r_{p}+\Delta_{x, \text { min }}$ ). The Level-Set function is fixed to $\phi=\phi_{d}$ and the difference between the drop volume and the VOF newly added is equally distributed to the VOF function on all cells up to the drop periphery, to ensure mass conservation. Finally, the redistance algorithm is used to regularize the function $\phi^{n}$ into the gas phase.

\subsubsection{Small droplets}

The potential collision of these droplets with an interface can happen in two different ways. In the first case, the droplet is considered as small even on the finest mesh, so that its mass and momentum can be directly injected into the local cell. In the second, the particle approaching the interface crosses one or more mesh refinement jumps: once its size exceeds the cell size, it is detected by the medium droplets search algorithm and switches in this category, so that it is projected on the Eulerian field before the actual impact (see paragraph 4.4.1).

\section{Results and validation}

In this section, the capabilities of the above developed method are presented. At first, dedicated test cases are provided to validate each step of the 


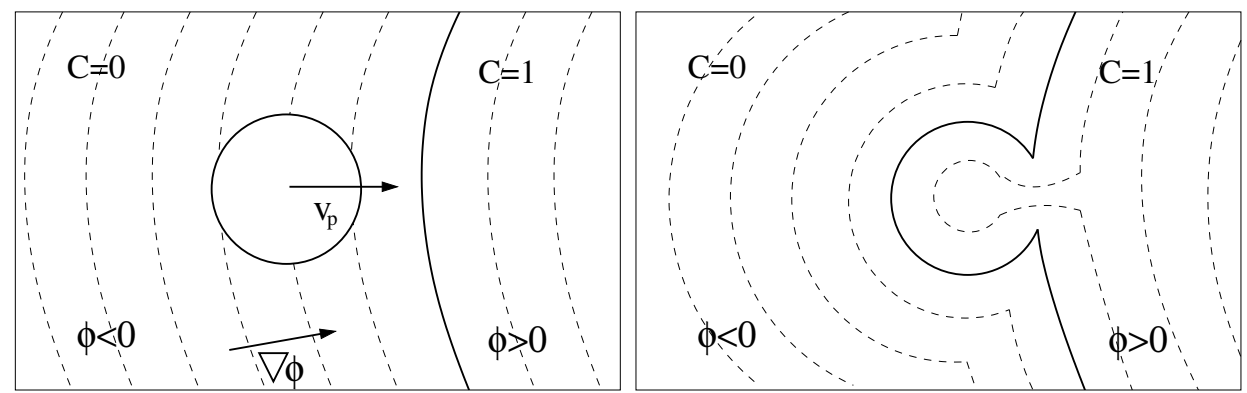

Figure 7: Illustration of the re-impact algorithm. Fat continuous line: interface $(\phi=$ $0, C=0.5)$, discontinuous line: iso-value of $\phi$. Left: before collision, right: after collision. When a particle is closed to the interface moving throw it, the re-impact is allowed: both VOF and Level-Set functions are reconstruct into the drop, and the Lagrangian particle is removed.

global algorithm; then, a more complex practical case consisting of a sheared liquid sheet atomization is shown.

\subsection{Flow accelerated drop}

This test case is devoted to the numerical verification of the medium droplet treatment. The depicted configuration consists of a spherical droplet sheared by an air flow. This is the typical scenario expected in the atomization droplet generation, where liquid structures are sheared and disintegrated, and the resulting droplets accelerated by drag forces. This situation corresponds to the one described in paragraph 4.1, where the droplet spans several cell size diameters and point-particles drag-force models cannot be employed. This test compares the solution given by a pure CLSVOF resolution versus the presented medium-droplet advection algorithm. In this test a very low density ratio has been considered in order to avoid any numerical method related error.

A three dimensional liquid drop initially at rest $\rho_{l}=2 \mathrm{~kg} \cdot \mathrm{m}^{-3}, \mu_{l}=$ $0.001 \mathrm{~kg} \cdot \mathrm{m}^{-1} \cdot \mathrm{s}^{-1}$ is immersed into an uniform gas stream $\rho_{g}=1 \mathrm{~kg} \cdot \mathrm{m}^{-3}$, $\mu_{g}=0.001 \mathrm{~kg} \cdot \mathrm{m}^{-1} \cdot \mathrm{s}^{-1}$ with constant velocity in the $x$ direction $\mathbf{u}(t=0)=$ $\left(U_{0}, 0,0\right)$ with $U_{0}=0.1 \mathrm{~m} . \mathrm{s}^{-1}$ and a surface tension $\sigma=0.001 \mathrm{Nm}^{-1}$. The computational domain consists of a square box of size $L=0.25 \mathrm{~m}$ with slip conditions on the walls tangent to the gas stream and inlet or outlet on the two walls orthogonal to the stream. This leads to the following non 
dimensional numbers, based on the drop radius $d_{d} / 2=r_{d}=0.05 \mathrm{~m}$ :

$$
W_{e}=\frac{\rho_{l} U_{0}^{2} r_{d}}{\sigma}=1, \quad R_{e}=\frac{\rho_{l} U_{0} r_{d}}{\mu_{l}}=5
$$

The test consists of the acceleration of the droplet up to a physical time of $t_{\text {end }}=2 \mathrm{~s}$. Several computations done with the CLSVOF with increasing refined meshes shows the behaviour of the Eulerian resolution, and the associated mesh convergence. Subsequently, a multi-scale treatment realized on the coarsest of the meshes compares the results obtained with the advanced "medium" droplet treatment. Four increasingly refined meshes have been considered, (M16, M32, M64, M128), with respectively $n_{\text {cells }} / L=$ $(16,32,64,128)$ corresponding to $n_{\text {cells }} / d_{p}=(3.2,6.4,12.8,25.6)$ cells per droplet diameter. Several Eulerian and two multi-scale medium droplet computations have been performed:

$\triangleright$ Three CLSVOF simulations on the three meshes [EUL_32], [EUL_64] and [EUL_128]

$\triangleright$ Two "medium droplet" simulations on the two meshes [LAG_16] and [LAG_32]

A point-particle drag-force model two-way Lagrangian computation has been performed as well on the M32 mesh. The particle is initialized as in the pure Eulerian case, but the multi-scale algorithm is allowed to detect and transform at the very first time-step: the Level-Set and VOF liquid fields are removed and a Lagrangian point particle is added with diameter equal to $d_{p}=0.1 \mathrm{~m}$.

Figures 8 and 9 show a direct comparison between the mesh converging Eulerian results, a point-particle two way drag force model and the Lagrangian medium droplet formulation. The visualization clearly shows that the final position of the droplet $x\left(t=t_{\text {end }}\right)$ is shifted downstream when a more accurate resolution is performed. The EUL_32 mesh droplet is late in comparison with the reference result of the EUL_128 mesh. The pointparticle two way drag force model used for the small particles accelerates too much the droplet, as the undisturbed velocity has been altered by the coupling term. Conversely, the Lagrangian result LAG_32 shows a good agreement with the most accurate EUL_64 and EUL_128 meshes. The velocity field obtained with the mixed formulation is close to the one obtained 

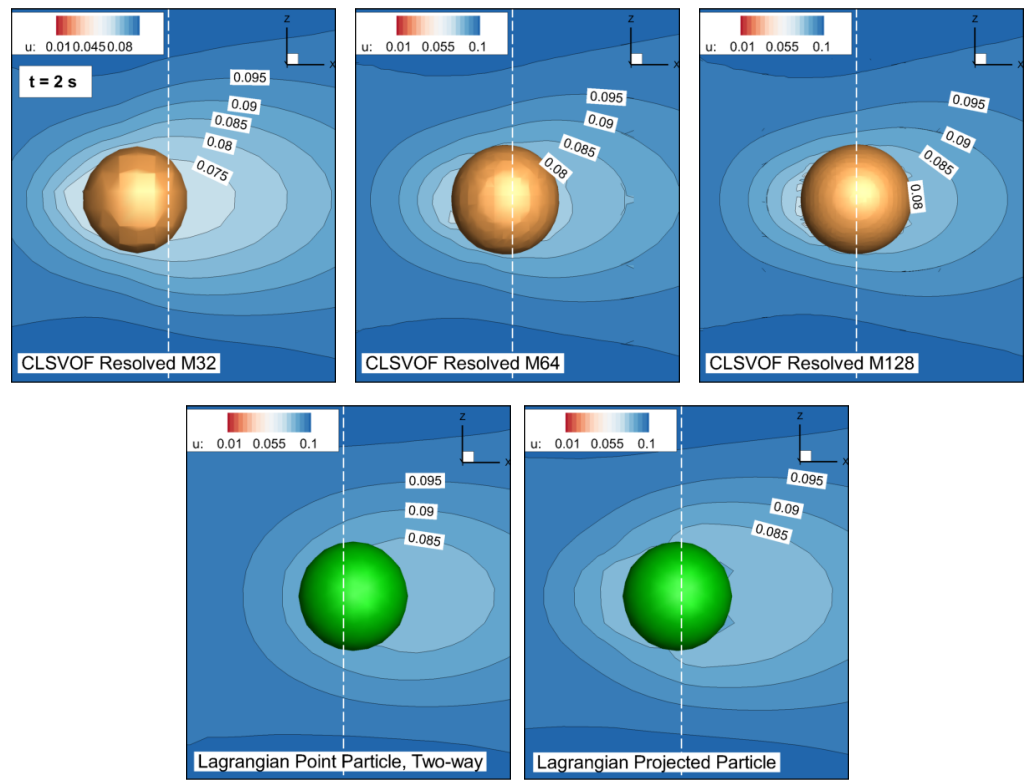

Figure 8: Advection of the liquid droplet at $t_{\text {end }}=2 \mathrm{~s}$. Upper line: from left to right, three CLSVOF results with increasing mesh refinement (EUL_32, EUL_64, EUL_128). Lower line: results of the point-particle two-way an the multi-scale computation LAG_32. The colour field is the $x$ (horizontal) component of the velocity $\mathbf{u}$. The vertical dotted white line represents the $x$ position of the droplet center of mass, as calculated with the EUL_128 simulation at $t=t_{\text {end }}$.

with the Eulerian resolution, whereas a point-source approximation fails to correctly impose the particle disturbance on the carrying flow. The position and velocity of the droplet have been measured, their values plotted against time in Figure 10. In the CLSVOF computation, the position of the center of mass and resulting velocity of the droplet have been evaluated as:

$$
\begin{aligned}
& \mathbf{x}_{\text {drop }}=\frac{\int_{\Omega_{l}} \mathbf{x} d v}{\int_{\Omega_{l}} d v}=\frac{\sum_{i} C_{i} \mathbf{x}_{i}}{\sum_{i} C_{i}} \\
& \mathbf{v}_{\text {drop }}=\frac{\int_{\Omega_{l}} \mathbf{u} d v}{\int_{\Omega_{l}} d v}=\frac{\sum_{i} C_{i} \mathbf{u}_{i}}{\sum_{i} C_{i}}
\end{aligned}
$$

In the Eulerian/Lagragian case, the point particle coordinate is directly available, as well as the advection velocity computed by (29) within the medium droplet algorithm. A Lagrangian computation with a very coarse mesh (M16) has been plotted as well. The plots clearly show how the Lagrangian medium 

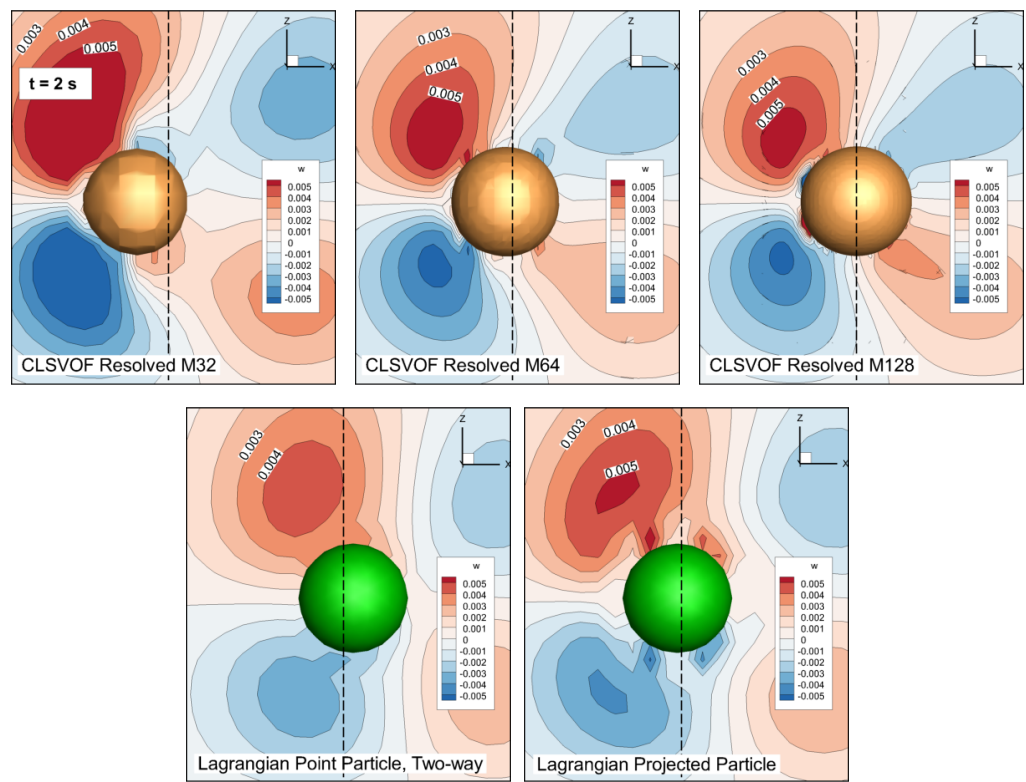

Figure 9: Advection of the liquid droplet at $t_{\text {end }}=2 \mathrm{~s}$. Upper line: from left to right, three CLSVOF results with increasing mesh refinement (EUL_32, EUL_64, EUL_128). Lower line: results of the point-particle two-way an the multi-scale computation LAG_32. The colour field is the $z$ (vertical) component of the velocity $\mathbf{u}$. The vertical dotted white line represents the $x$ position of the droplet center of mass, as calculated with the EUL_128 simulation at $t=t_{\text {end }}$.

droplet modelling is able to reach almost the same accuracy as the Eulerian modelling with a mesh size twice as refined. It is also visible how the inaccurate prediction of the increasing velocity for the EUL_32 solution is affected by an initial plateau between $t=0 s$ and $t=0.5 \mathrm{~s}$ : the initial acceleration being delayed, the following evolution is shifted towards lower values.

\subsection{Settling drop}

In this section the numerical resolution of a medium droplet settling in a large tank of stationary gas is assessed. The test is taken from [41], where the droplet is modelled by a point particle drag force approach with a special treatment for the undisturbed velocity. In this test a higher density ratio of 100 has been considered. The low droplet Reynolds number allows to test the particle-altered viscous effects as well.

The droplet is initially placed in the upper half of the tank. Gravity accelerates the particle up to the settling velocity, where equilibrium is 


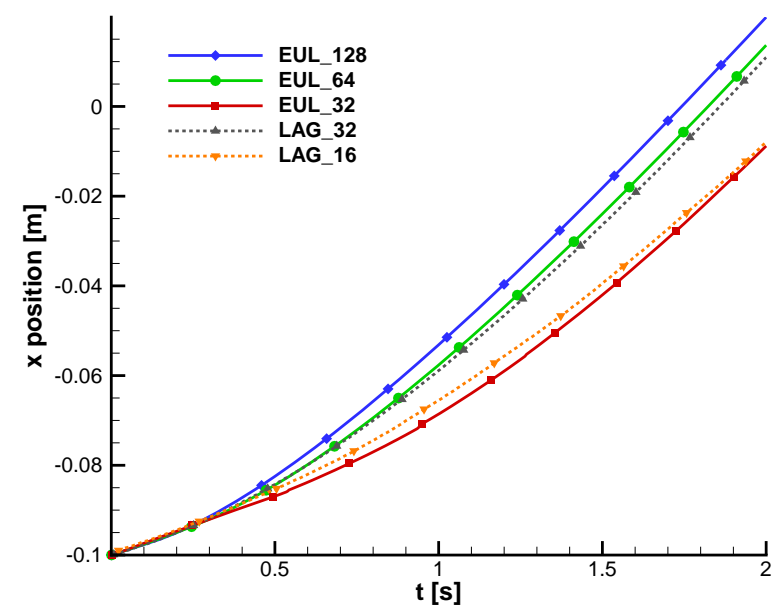

(a)

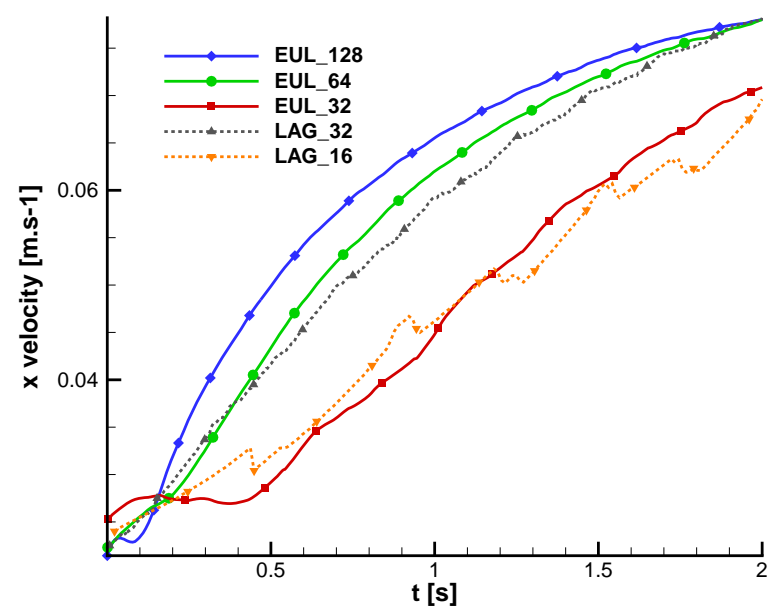

(b)

Figure 10: (a) $x\left(t=t_{\text {end }}\right)$ position of the droplet (b) $x$ velocity component of the droplet versus time. Continuous lines: Eulerian computation, dotted lines: Lagragian mediumdroplet. 
reached with the drag force. The dimensions of the tank are $\left(L_{x}, L_{y}, L_{z}\right)=$ $(1,1,2) \mathrm{mm}$. The drop is initially placed at $\mathbf{x}_{d}\left(t=t_{0}\right)=\left(0.5 L_{x}, 1.5 L_{y}, 0.5 L_{z}\right)$, its diameter being $d_{d}=L_{x} / 10=0.1 \mathrm{~mm}$. The liquid droplet parameters are $\left.\rho_{l}=1000 \mathrm{~kg} \cdot \mathrm{m}^{-3}, \mu_{l}=10^{-3} \mathrm{~kg} \cdot \mathrm{m}^{-1} \cdot \mathrm{s}^{-1}\right)$, the gas $\rho_{g}=10 \mathrm{~kg} \cdot \mathrm{m}^{-3}$, $\left.\mu_{g}=10^{-4} \mathrm{~kg} \cdot \mathrm{m}^{-1} \cdot \mathrm{s}^{-1}\right)$. Following [41], the predicted settling velocity is $v_{t} \approx 0.04 \mathrm{~m}^{-1} \cdot \mathrm{s}^{-1}$, reached at about $t \approx 0.03 \mathrm{~s}$. The Reynolds number based on the terminal velocity is $R e_{t} \approx 0.04$. At this low $R e$ the viscous effects are strong enough to keep the droplet spherical: no surface tension is considered. In this test the limits of the pure Eulerian and Lagrangian approaches described in the introduction can be clearly investigated. Those limits concern in particular the mesh cell size compared to the droplet diameter.

$\triangleright$ The Eulerian CLSVOF computation should need a certain number of cells into the droplet to effectively resolve it. One can expect poor quality results when approaching $\Delta x \approx d_{d}$.

$\triangleright$ The pure point-particle Lagrangian solution (the two-way approach) should conversely behave correctly when $\Delta x>>d_{d}$. When $\Delta x \approx d_{d}$, the momentum source term is entirely applied on the cell containing the particle. This would in turn alter the undisturbed velocity seen by the particle, thus perturbing the computation of the drag force. Moreover, the cells surrounding the concerned one can not develop a correct Eulerian field as no influence comes from the particle itself.

In order to assess these statements, a certain number of simulations have been performed, the aim being to measure the settling. A base mesh of $(n x, n y, n z)=(24,24,48)$ cells $(\mathrm{M} 24)$ has been chosen, as the droplet diameter is exactly 2,4 cells, a pertinent value for the multi-scale approach assessment. The simulations include:

$\triangleright$ An one-way point-particle simulation, on M24 (this is mesh independent, however) [LAG_24_1W]

$\triangleright$ A two-way point-particle simulation, on M24 [LAG_24_2W]

$\triangleright$ Three CLSVOF pure Eulerian simulations on increasing refined meshes: M24, M48 and M96, for mesh convergence purpose [EUL_XX]

$\triangleright$ A multi-scale "medium particle" simulation on M24 [LAG_24_MP] 
The meshes M48 and M96 correspond to 48 and 96 mesh cells in the $x$ direction. The droplet resolution in the three meshes is respectively $n_{\text {cells }} / d_{d}=$ $(2.4,4.8,9.6)$. The particle is always initialized as in the Eulerian case; in the Lagrangian computations the multi-scale algorithm is allowed to detect and transform at the very first time-step: the Level-Set and VOF liquid fields are removed and a particle is added with diameter equal to $d_{p}=0.1 \mathrm{~m}$.

In this simple scenario, a theoretical terminal velocity can be estimated in the Stokes flow hypothesis. At this velocity, the Stoke's drag is balanced by the gravity force acting on the particle:

$$
F_{d}=6 \pi \mu r v_{t}=m_{p} g=\frac{4}{3} \pi\left(\rho_{l}-\rho_{g}\right) g
$$

Solving for $v_{t}$ gives:

$$
v_{t}=\frac{2 r_{p}^{2}\left(\rho_{l}-\rho_{g}\right) g}{9 \mu_{g}}
$$

In 41 it has been found that the relative small size of the computational domain induce confinement effects, effectively decreasing the terminal velocity. A correction of the classical Naumann-Schiller formulation (32) can be found in [43]:

$$
\tau_{p}=\tau_{p}^{S N} \eta=\tau_{p}^{S N}\left(\frac{1-d_{p} / L_{x}}{1-0.33 d_{p} / L x}\right)^{2.7}
$$

The corrected predicted terminal velocity would then be

$$
v_{t} \approx 0.04468 m . s^{-1}
$$

On the other hand, a numerical reference solution can be easily obtained by the point-particle drag-force computation in a one-way approach: as the theoretical undisturbed velocity is zero, the drag force models always sees the zero initial condition as an input. In this case the simulation is actually mesh independent. Figure 11 shows instantaneous results from three simulations at $t \approx 0.02 \mathrm{~s}$, in the order EUL_96, LAG_24_MP and LAG_24_2W. The contour field corresponds to the vertical velocity $v_{y}$, the vectors to the local velocity vector. The images clearly suggest that a two-way point-particle approach induces an excessive acceleration of the particle. The surrounding velocity field is badly captured as well.

The "medium particle" approach, on the other side, tries to solve the two problems at once. The projection of the droplet information (equations (26), 
(27), 25) ) on all the concerned cells (equation (21)) allows the solver to build more correctly the surrounding gas flow. Given the low Reynolds number, the particle is not supposed to deform: the imposition of the spherical shape allows the simulation on a very coarse mesh to match CLSVOF results on a twice refined mesh.

Figure 12 shows the evolution of the droplet vertical velocity for the six different simulations. Using the one-way point-particle simulation (LAG_24_1W) as a reference solution, as explained beforehand, the worst result is obtained by the two-way approach (LAG_24_2W). The alteration of the cell velocity induced by the source term induces a large error in the drag force computation in equation (32). In the Eulerian results (EUL), the velocity plot shows a convergence towards a settling velocity for the most refined meshes. The coarsest mesh M24 has however troubles finding a steady-state solution, and a noticeable overshoot of the reference solution is visible between 5 and 20 ms. It is noticeable a slight drop deformation which can explain the difference between these simulations and the reference solution. The "medium particle" simulation agrees very well with the reference solution, even if mesh-related oscillations appear all along the computation.

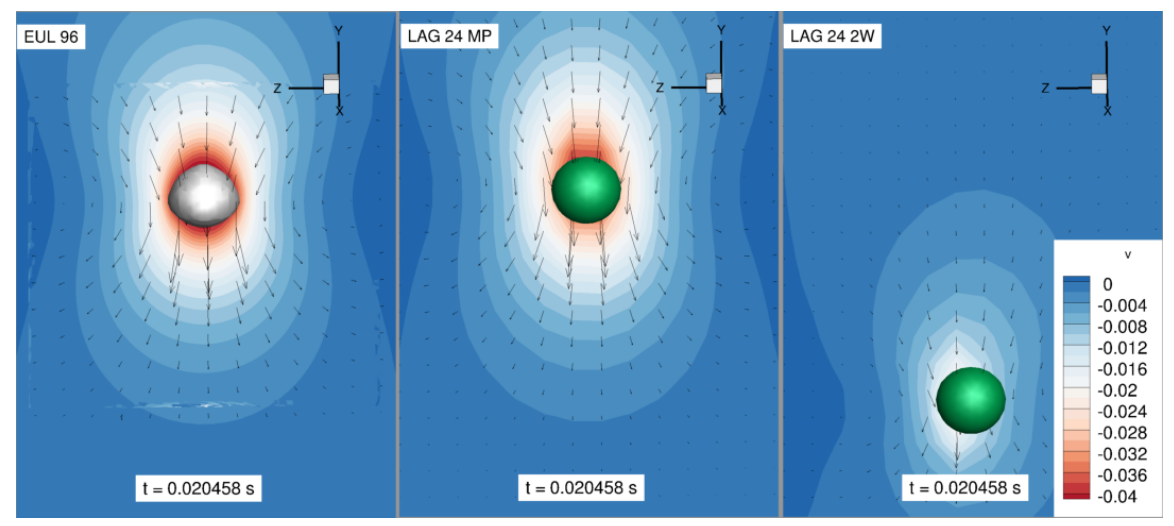

Figure 11: Droplet falling velocity for different simulations.

Table 1 shows the settling velocity obtained by time averaging the $y$ direction component between 0.025 and $0.03 \mathrm{~s}$. The quantitative solutions confirm the previous results analysis. Figure 13 traces the $y$-position of the particle center of mass. As result of the different velocities computed in each simulation, the two-way simulation overestimates the droplet position, as it does the Eulerian coarsest mesh; the more refined Eulerian results are in 


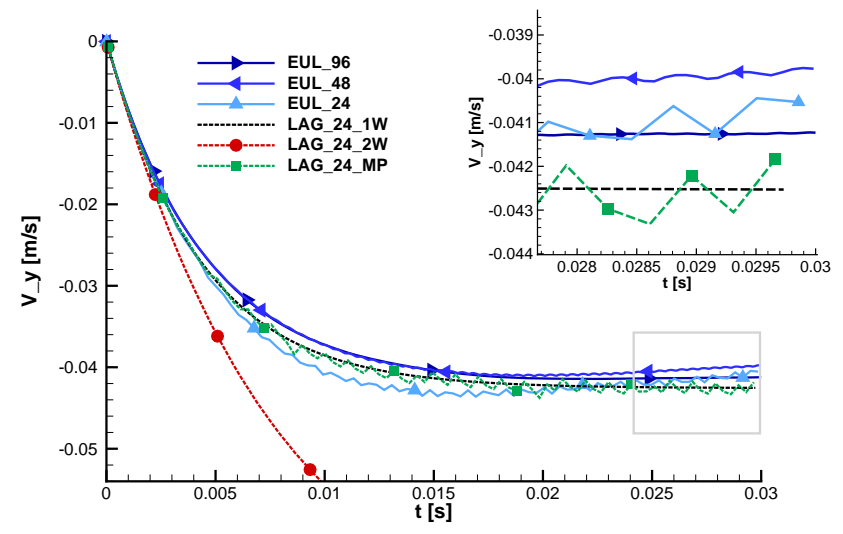

Figure 12: Droplet falling velocity for different simulations.

good agreement and differ of about $2 \%$. The medium droplet simulation follows very closely the reference solution. In this test the "medium particle" approach has shown to be indeed very effective.

\subsection{Drop-free surface collision}

In this section, the numerical procedure is experienced with a drop colliding a free surface at rest by comparing Eulerian and mixed Eulerian/Lagrangian computation. A water drop of radius $r=0.176 \mathrm{~m}, \rho_{l}=1000 \mathrm{~kg} \cdot \mathrm{m}^{-3}$, $\mu_{l}=10^{-3} \mathrm{~kg} \cdot \mathrm{m}^{-1} \cdot \mathrm{s}^{-1}$ initially at rest is immersed in air $\rho_{g}=1.225 \mathrm{~kg} \cdot \mathrm{m}^{-3}$, $\mu_{g}=10^{-5} \mathrm{~kg} \cdot \mathrm{m}^{-1} \cdot \mathrm{s}^{-1}$, inside a cubic domain of size $L=1 \mathrm{~m}$. The droplet is suspended over a free liquid surface placed at $y=0.25 \mathrm{~m}$; the initial position of the droplet is $\mathbf{x}_{d}\left(t=t_{0}\right)=(0.25,0.25,0.75) \mathrm{m}$ (the offset from the center of the domain is to avoid the particle crossing an interface between mesh blocks). A gravity field is imposed as $\mathbf{g}=(0,-9.81,0) m \cdot s^{-1}$. The droplet is let to accelerate under the gravity effect, until impact on the surface. This test is meant to test both the conversion and transport of medium drops algorithm as well as the particle re-impact.

As for the previous example, the domain is discretized with increasing fine meshes for both the pure Eulerian and the Eulerian/Lagrangian "medium particle" model. The meshes are M16, M32, M64. For the Lagrangian computation, the algorithm detects and transforms the droplet at the very first time-step. No slip conditions are applied everywhere. Five simulations are presented: 


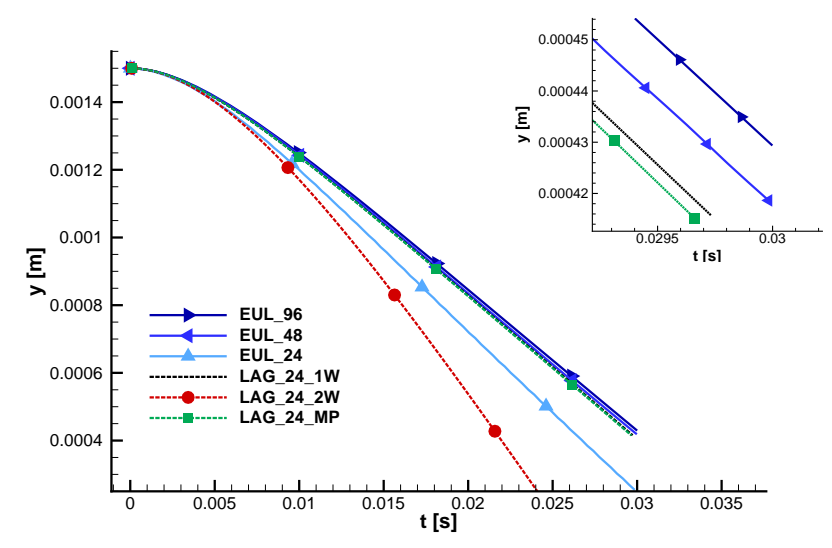

Figure 13: Droplet falling velocity for different simulations.

$\triangleright$ Three CLSVOF pure Eulerian simulations on increasing refined meshes M16, M32, M64, for mesh convergence purpose [EUL_XX].

$\triangleright$ Two multi-scale "medium particle" simulations on M16 and M32 [LAG_XX].

Figure 14 shows the results of the fall and impact of the droplet up to $t_{\text {end }}=2 s$, given by pure CLSVOF computation with the finest M64 mesh. At $t \simeq 0.35 \mathrm{~s}$ the droplet impacts the surface (the exact time depends on the mesh size). Circular surface waves develop after the impact, and a first geyser forms and reaches its peak at around $t \simeq 1.3 \mathrm{~s}$. The height of the rising geyser gives a good measure of the momentum exchange between the falling droplet and the surface. Figure 15 illustrates a direct comparison between the CLSVOF and the multi-scale resolution of the impact realized on the same M32 mesh (the finest mesh in which the droplet can still be transformed into Lagrangian medium particle). The results show a very good agreement between the two methods: in particular, the time of the impact is almost the same, and the dynamics of the surface waves and the rising geyser are almost identical. This means that a good droplet tracking has been performed, and that the Lagrangian to Eulerian conversion is conservative in mass and momentum. Figure 16 shows the maximum and minimum of the interface position, tracking the geyser and the sink after the impact. It can clearly be seen how the Lagrangian solution is better than the pure Eulerian one as the drop becomes less resolved. A small overshoot can be distinguished in the well, possibly due to the droplet keeping rigid until the activation of 


\begin{tabular}{r|cc}
\hline & $V_{t}\left[\mathrm{~m}^{-1} \mathrm{~s}^{-1}\right]$ & Difference \\
\hline Eq. $(47)$ & 0.04468 & - \\
\hline LAG_24_1W & 0.04233 & ref \\
\hline LAG_24_2W & $>0.07$ & $70 \%$ \\
\hline EUL_24 & $\approx 0.04185(\mathbf{n c})$ & - \\
\hline EUL_48 & 0.04047 & $4.4 \%$ \\
\hline EUL_96 & 0.04143 & $2.1 \%$ \\
\hline LAG_24_MP & 0.04262 & $0.68 \%$ \\
\hline
\end{tabular}

Table 1: Settling velocities of the falling droplet computed by numerical simulations. nc means that the velocity has not yet converged.

the re-impact algorithm, while the resolved drop start its deformation a little sooner. Tests with a point-particle drag-force model have clearly shown how the classical dispersed phase approach is not adapted in this configuration.

\subsection{Assisted atomization of a liquid sheet}

The whole multi-scale algorithm has been applied to the simulation of the primary atomization of a liquid sheet sheared by two parallel air streams. In this configuration, the sheet-shape is unstable. Hydrodynamic instabilities are the source of the atomization mechanisms and determine the primary break-up characteristics. During the atomization process, two different stages can be distinguished [1], 2], 3], 4]. In the primary atomization the sheet becomes subject to longitudinal instabilities, which are the results of the shearing effect: Kelvin-Helmholtz instabilities perturb the plane sheet, starting a sinusoidal streamwise oscillation all along the sheet. Then, fully three dimensional instabilities generate transverse modulations. The sheet breaks into smaller liquid packs, ligaments and bag-like structures. This continuous fragmentation ends with the formation of a polydisperse spray of droplets.

The considered numerical configuration aims to reproduce a simple atomization device as the one experimentally investigated in [44]. The injector, depicted in Figure 17(a), consists of a NACA-shaped injector immersed into a channel gas flow, discharging the liquid by a rectangular fissure $300 \mu m$ thick (visible in the middle of the device nozzle). Different geometrical configurations as well as liquid and gas flow regimes are considered by the authors. In 


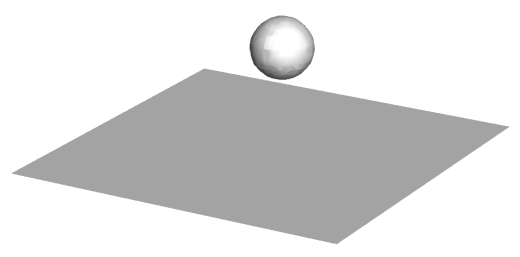

(a) $t=0.00 \mathrm{~s}$

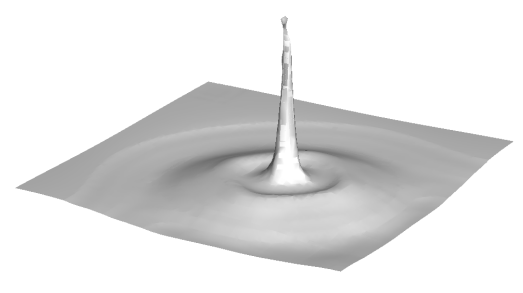

(c) $t=1.25 \mathrm{~s}$

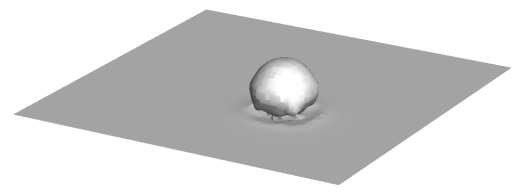

(b) $t=0.35 \mathrm{~s}$

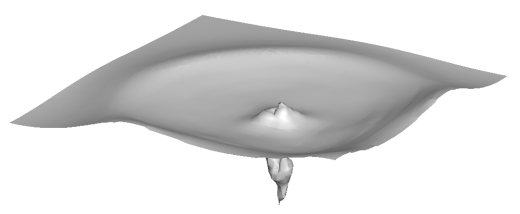

(d) $t=1.75 \mathrm{~s}$

Figure 14: Results of the CLSVOF computation of a droplet splashing on a liquid free surface, resolution $64^{3}$.

this work a liquid sheet maintaining the same non dimensional parameters $\mathrm{M}, \mathrm{Re}$, We (momentum flux ratio, gaseous Reynolds and Weber ) has been simulated. A ratio of 100 has been imposed between the liquid and gas density, as a ratio of 1000 has shown to produce numerical instabilities leading to non-convergence in presence of strong shearing flows. The chosen physical parameters of the simulation are typical of a "stretched ligament" break-up, where the primary atomization manifests in the form of membranes and longitudinal ligaments formation. The momentum ratio involving the "stretched ligament" break-up are $0.5<M<4$ (from [4] and [45]).

The parameters are summarized in table 2 . The simulation domain, with reference to Figure 17(b), is a rectangular box of $L_{x}=40.96 \mathrm{~mm}, L_{y}=10.24$ $\mathrm{mm}, L_{z}=5.12 \mathrm{~mm}$. AMR blocks of $(n x, n y, n z)=(16,16,8)$ cells have been used; a total of 5 levels of refinement give an equivalent fine mesh of $(n x, n y, n z)=(1024,256,128)$, which means $\Delta x=40 \mu m$. Inflow condition are imposed on the left face, slip conditions on the horizontal and vertical 

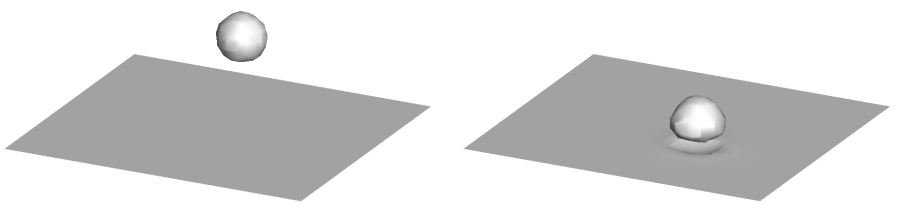

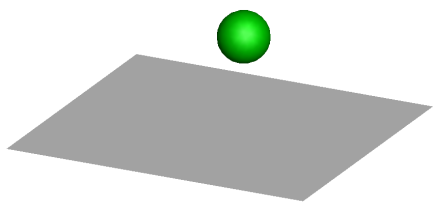

(a) $t \simeq 0.00 \mathrm{~s}$
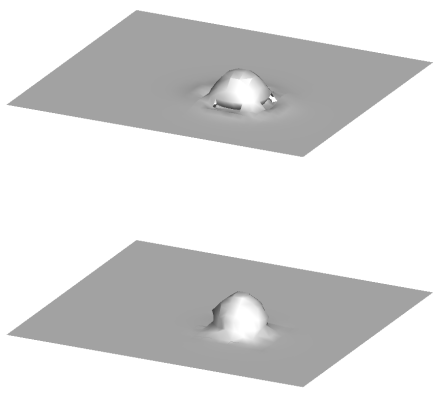

(c) $t \simeq 0.38 \mathrm{~s}$
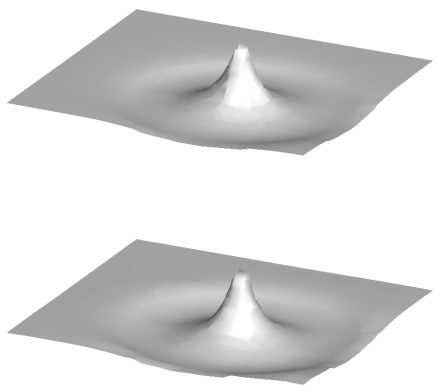

(e) $t \simeq 1.00 \mathrm{~s}$

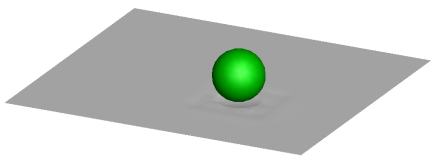

(b) $t \simeq 0.35 \mathrm{~s}$
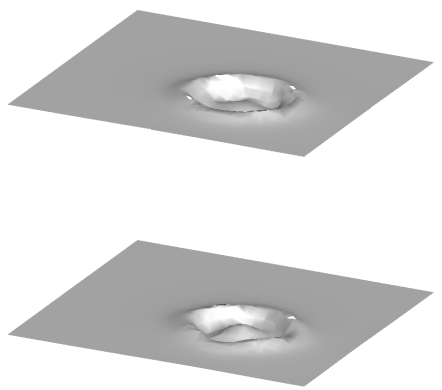

(d) $t \simeq 0.45 \mathrm{~s}$
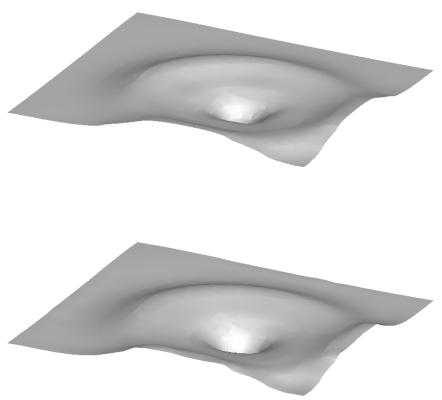

(f) $t \simeq 1.50 \mathrm{~s}$

Figure 15: Visual comparison between the CLSVOF (upper image) and the multi-scale resolution (lower image) of a droplet splashing on a liquid free surface, resolution $32^{3}$. 


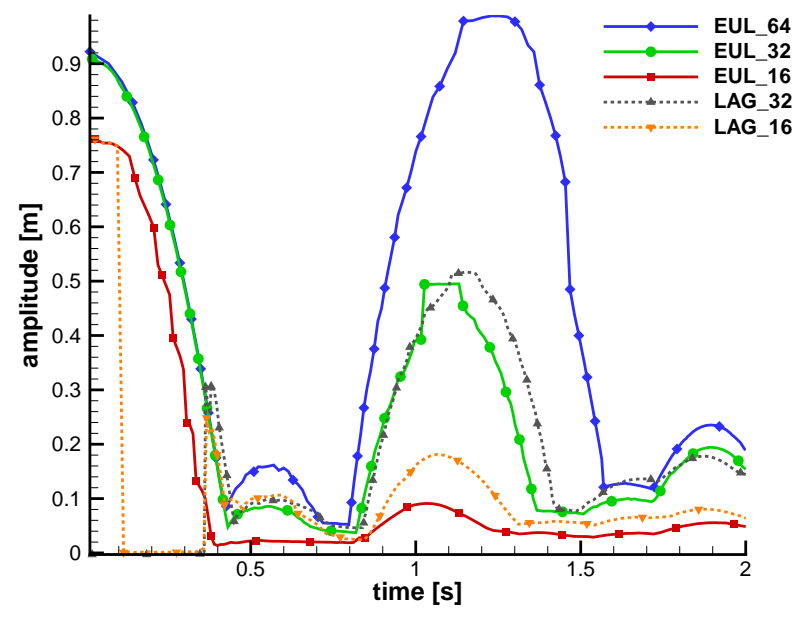

(a) Maximal amplitude

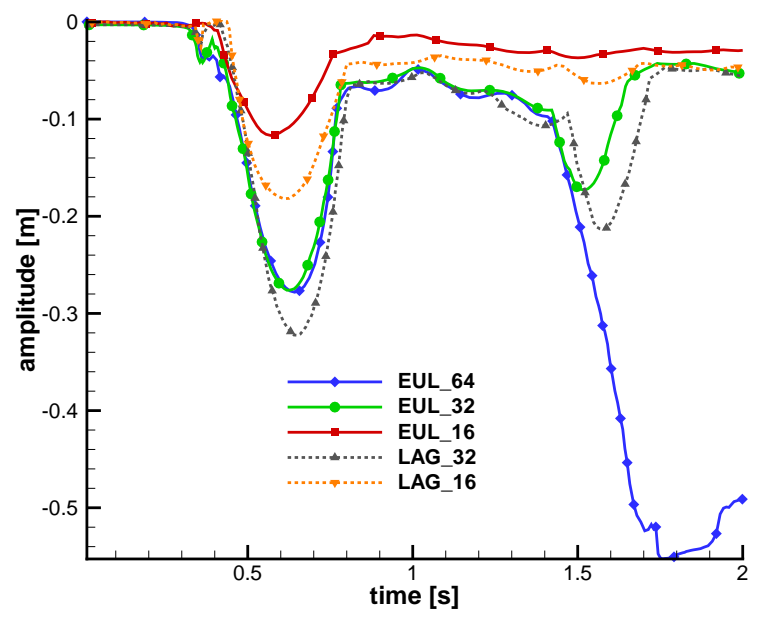

(b) Minimal amplitude

Figure 16: Maximal and minimal positions of the interface in the $y$ direction (along the trajectory of the droplet). The maximal amplitude shows the primary geyser after the impact, the minimal the depth of the imprint. 


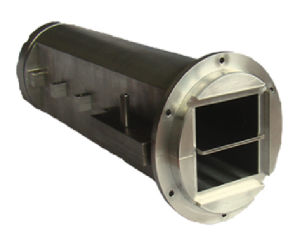

(a)

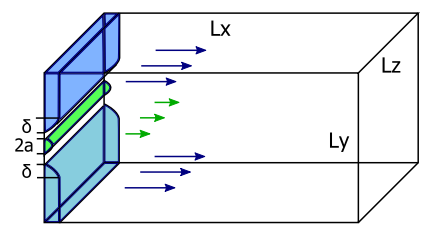

(b)

Figure 17: (a) Actual liquid sheet injector device. (b) Numerical computation set-up.

\begin{tabular}{llr}
\hline Geometrical parameters & & \\
\hline Sheet thickness & $2 a$ & $300 \mu m$ \\
Boundary layer thickness & $\delta$ & $270 \mu m$ \\
\hline Non-dimensional parameters & 1115 \\
\hline Gas Reynolds & $R e_{g}=\frac{\rho_{g} u_{g} \delta}{\mu_{g}}$ & 104 \\
Liquid Reynolds & $R e_{l}=\frac{\rho_{l} u_{l} a}{\mu_{l}}$ & 17.42 \\
Weber & $W e=\frac{\rho_{g}\left(u_{g}-u_{l}\right)^{2} a}{\rho_{g} u_{g}^{2}}$ & 0.91 \\
\hline Momentum ratio & $M=\frac{\rho_{l} u_{l}^{2}}{2}$ & \\
\hline
\end{tabular}

Table 2: Geometric and dimensionless parameters of the 3D atomization simulation.

faces and outflow on the right face. The droplet detection algorithm is capped at a maximum droplet size of eight $\Delta x$, giving a possible diameter range of [0:320] $\mu \mathrm{m}$. Figure 18 shows the initial destabilization of the liquid sheet. In Figure 18(a) the longitudinal instability starts to develop. Hints of the transverse instabilities are visible as well. In Figure 18(b) the instabilities are further developing, and liquid packs resulting from the first sheet break-up have detached from the sheet main body. Ligaments have begun to form under the three-dimensional instabilities effects. Droplets begin to detach from the ligaments and the membranes, their size already varying from the smallest to the largest detectable by the algorithm. Figure 18(c) shows the sheet atomization established regime, with the continuous generation of liquid blobs and small droplets. Many large liquid structures keep stable up to the exit of the numerical domain. A variety of droplet diameters are found 
by the detection algorithm. Figure 19 zooms to the mechanism of ligament break-up: the ligament is stretched by a Rayleigh-Plateau instability up to the detachment of a satellite droplet, which is immediately transformed into a Lagrangian point particle. It is visible how the droplet moves to a less refined zone, far from the resolved sheet, as it is expected from the multi-scale algorithm.

A comparison of some large scale features of the atomization process has been made with respect to the experimental results found in [44], in terms of visual aspect, oscillation frequency and mean break-up length. A visualization of the behaviour of the sheet is given in Figure 20. The simulation correctly captures the growing longitudinal instability of the sheet, as well as the transversal modulation. The thin ligaments formed by the primary atomization at the break-up point are fully captured by the CLSVOF eulerian solution. A first wave of droplets is formed at this location. The liquid detached from the main sheet body undergoes further deformation and break-up into a fully three-dimensional flow: it can be seen in both images that large liquid parcels coexist with smaller stable droplets. The oscillation frequency of the simulation (table 3 ) is quite higher than the measured one, while the breakup length is underestimated. This seems to be a recurrent problem in two phase DNS simulations of liquid sheet atomization ([46], [47], [36], [48]). Possibly, the scale in the physical parameters of the simulation should be somehow taken in account in the frequency computation. The authors could not find however any pertinent scaling factor.

\begin{tabular}{rcc}
\hline & Experience & Simulation \\
\hline Oscillation frequency $[\mathrm{Hz}]$ & 438 & 880 \\
\hline Break-up length $[\mathrm{mm}]$ & 9.8 & 6.46 \\
\hline
\end{tabular}

Table 3: Quantitative liquid sheet characteristics, simulation and experience.

A preliminary analysis has been performed on the droplet spray, the results compared to the experience by laser diffraction system allowing measurement of spray droplet size distributions. The statistics involve the droplets in the whole numerical domain; their characteristics are averaged in time, the samplings taken with a frequency corresponding to an average convection time in order to avoid repeated recording of the same droplet. The particles of diameter inferior to the finest mesh cell $(40 \mu \mathrm{m})$ have been discarded, as 

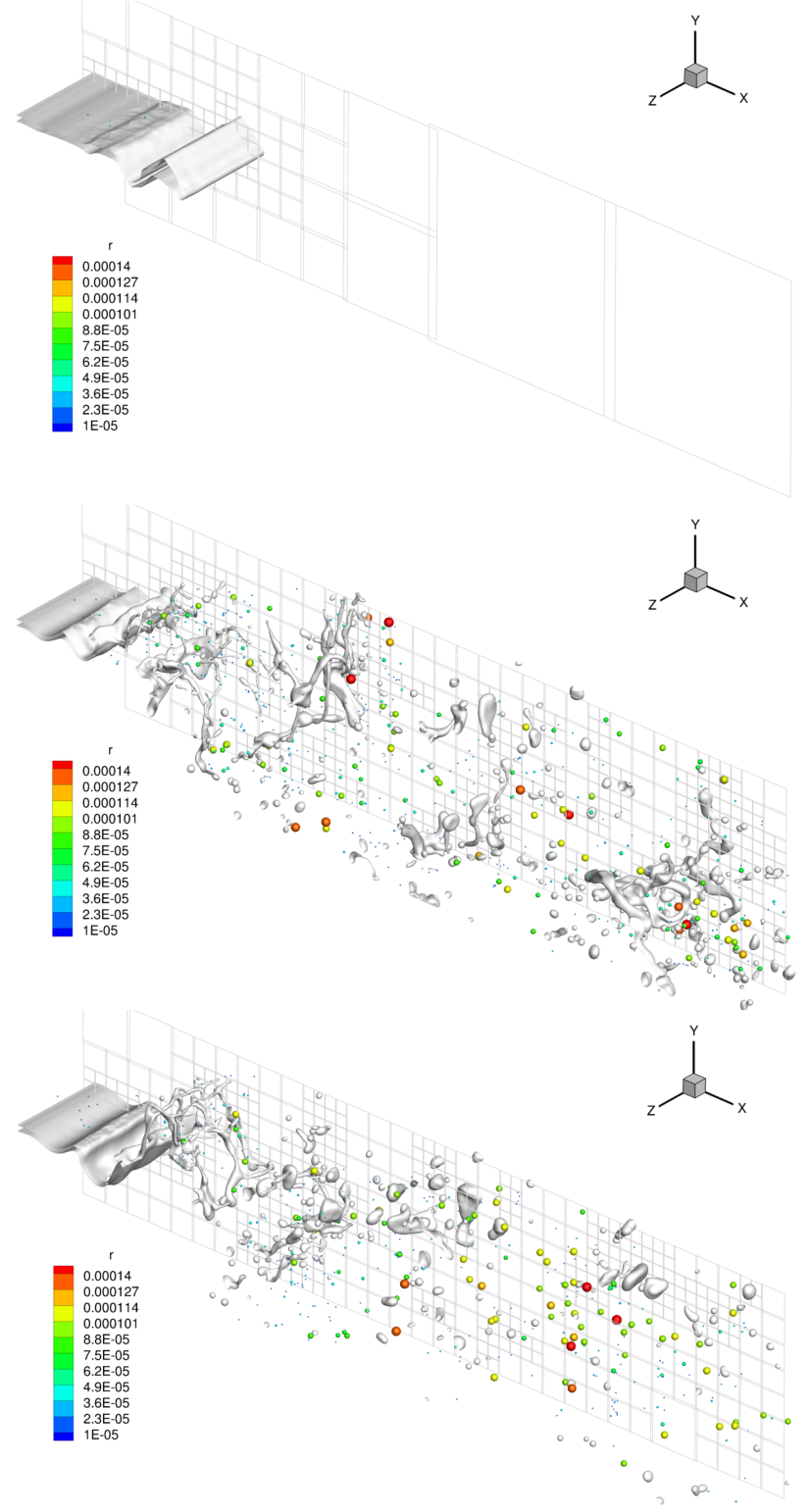

(c)

Figure 18: Results of the planar liquid sheet atomization. (a) Initial destabilization (b) Fully developed atomization. Coloured spheres: Lagrangian particles (both small and medium ones), actual size, coloured by radius. 


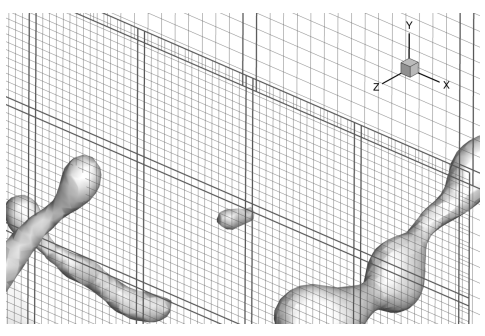

(a)

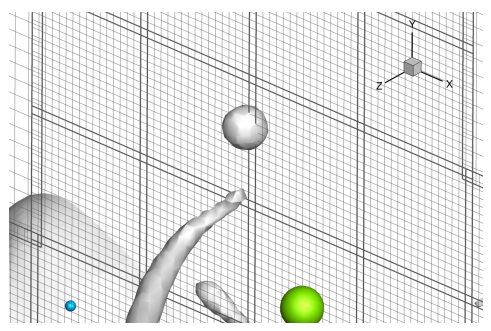

(c)

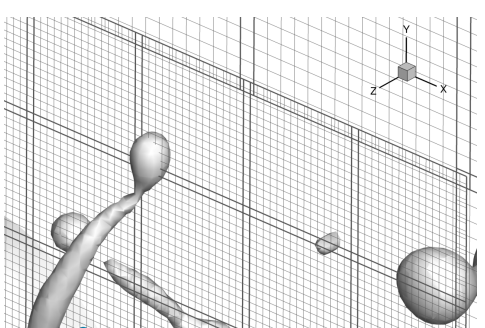

(b)

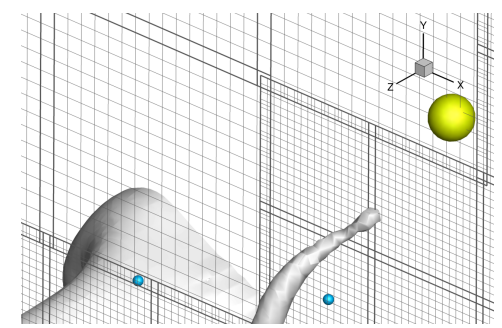

(d)

Figure 19: Capture of the generation of a droplet: Rayleigh-Plateau instability, ligament stretching, break-up and Eulerian to Lagrangian transition.

their formation is dominated by numerical effects. Their mass can still be kept in account for liquid mass flows if needed.

A total of 18457 droplets $\left(40<d_{p}<320 \mu m\right)$ have been registered. Figure 21 shows the corresponding distribution in volume. The simulation seems able to capture the predominant diameters around 220-250 microns. No information has been extracted from structures larger than 320 microns in diameter, so that the distribution is truncated after this value. Droplets smaller than the finest mesh size have been ignored as well. A comparison with the ONERA experience shows similarities in the volume distribution. The experimental distribution has been truncated in the same way, so that both distributions are equally normalized. However, the experimental diameter measurements have been performed far outside the computational domain of the simulation. The simulated cloud of droplets could probably undergo secondary atomization if much larger domain could be simulated. One of the key feature of the DNS is the ability to measure relevant quantities inside the very dense primary atomization region. 

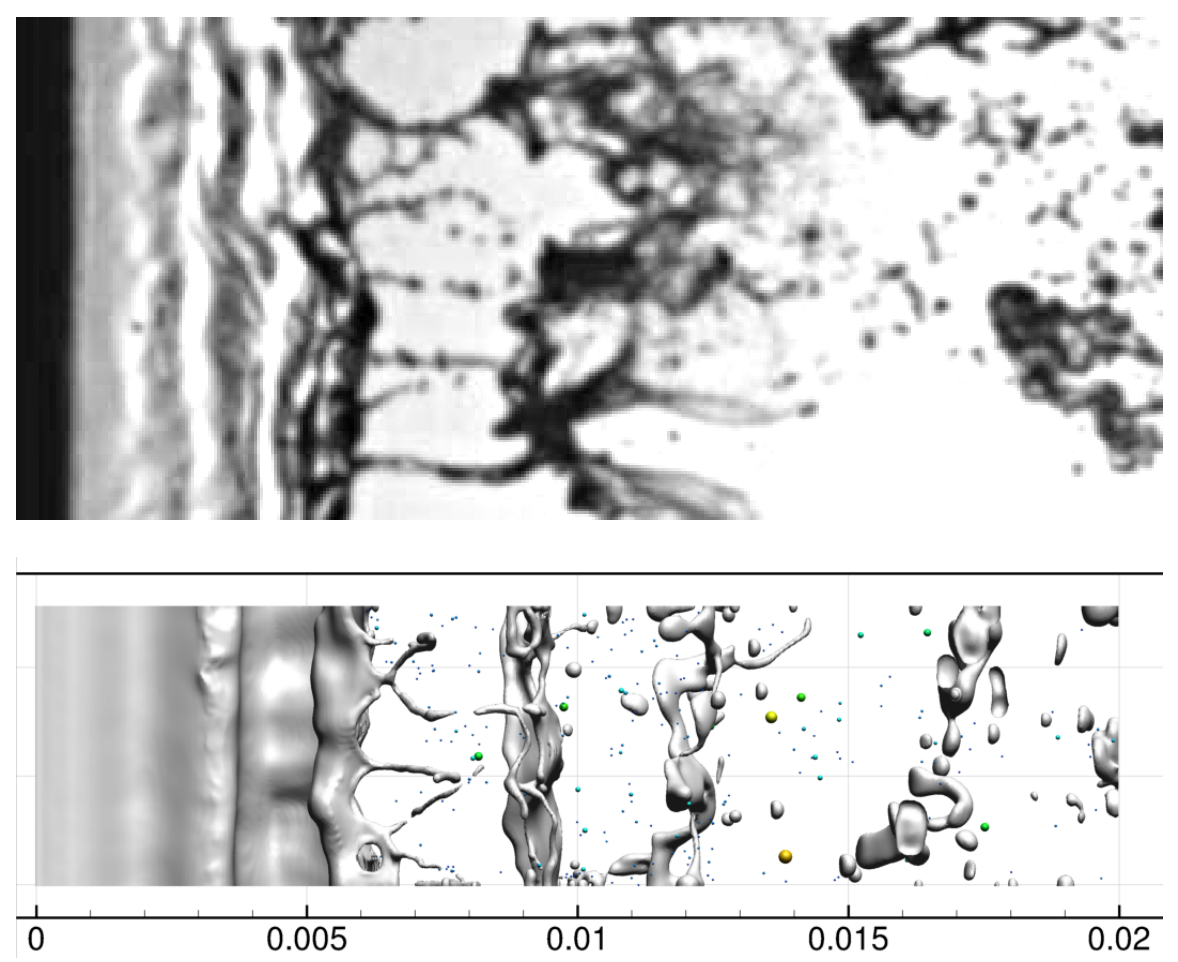

Figure 20: Snapshots of both experimental visualization and simulation, arbitrary time step.

\subsection{Computational cost analysis}

The multi-scale approach described in this paper adds a certain number of operations to the standard CLSVOF algorithm. In this section an estimate of the cost of the algorithm is given. The estimation is based on a fixed number of time steps performed on the previously presented simulation, section 5.4 . Timers have been added to the code in pertinent locations, by means of the MPI function MPI_WTIME(). The timings of the following three steps performed at each time-step have been recorded:

1) The interface tracking (section 3 , equations $(9)$ and (5)), including most of the multi-scale algorithm (section 4)

2) The momentum update (section 3 , equation (11))

3) The pressure equation (section 3 , equation (11) 


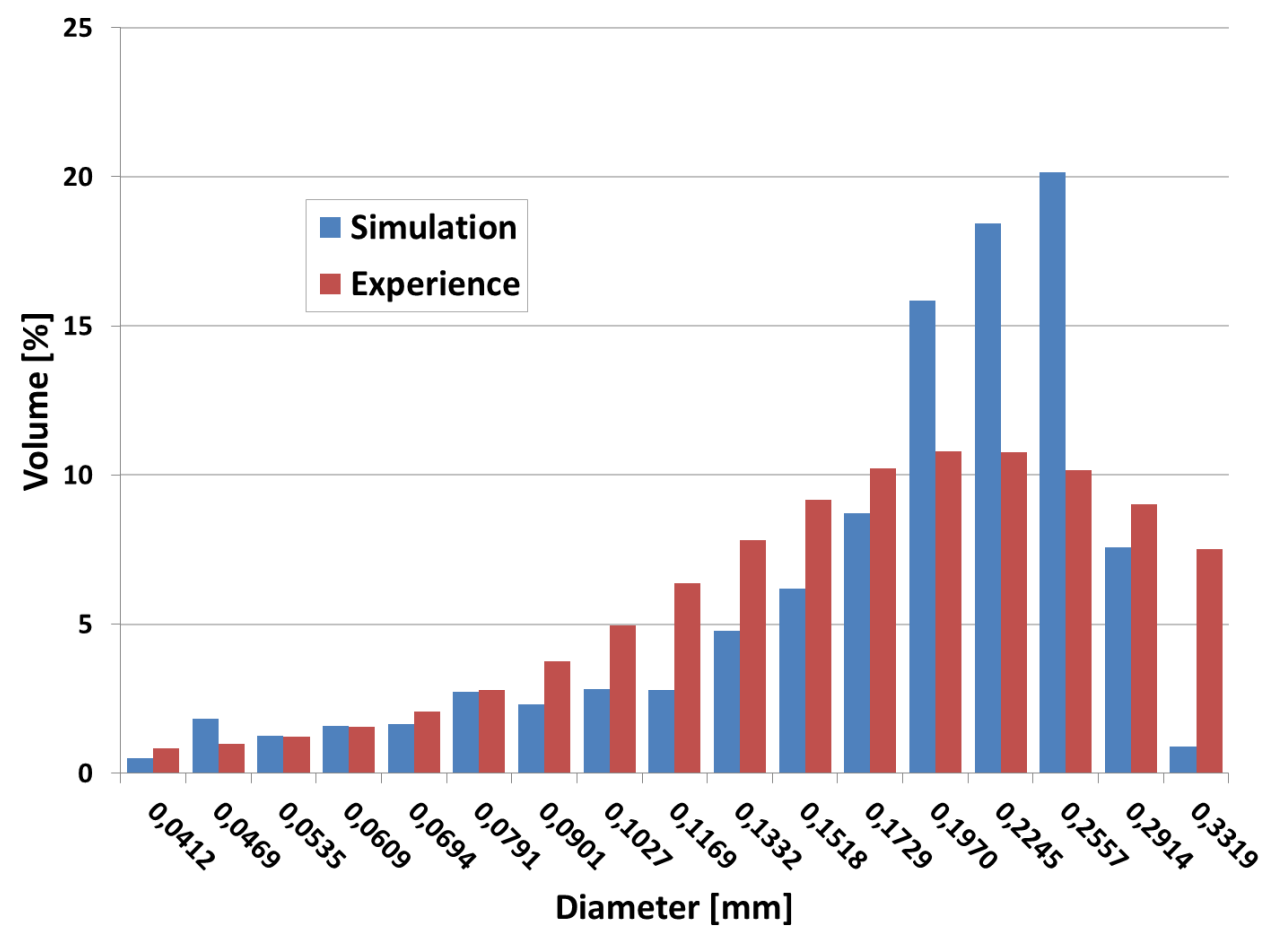

Figure 21: Droplet volume distribution obtained from the simulation dispersed phase, comparison with the experience.

However, these results should be taken as a qualitative estimation of the actual computational cost, for the following reasons:

$\triangleright$ The pressure solver may converge in a different number of iterations, depending on the mesh and the fluid topology and characteristics.

$\triangleright$ The CLSVOF interface reconstruction is active in the mixed cells only, meaning that its computational cost is linearly depending on the total density of interface (i.e. the ratio between mixed and full/empty cells).

$\triangleright$ The sheer number of droplets detected and treated by the multi-scale algorithm is obviously problem-dependent.

A relevant parameter of the computations is the number of cells in each AMR block. This value is however a constant for a given computation, the value $(n x, n y, n z)=(16,16,16)$ having been retained as an optimal value. Another "free" parameter is the frequency of the tagging algorithm action 
(paragraph 4.1). This is probably the most expensive part of the EulerLagrange algorithm. It is currently called every 10 time steps, a value giving ample time to detect the liquid structures given the CFL condition. It may be probably possible to further decrease this frequency. In addition, the liquid detection is supposed to act on the smallest AMR level only, thus drastically reducing the total volume to be analysed. Table 4 presents the

\begin{tabular}{cc}
\hline \multicolumn{2}{c}{ Normalized time } \\
\hline CLSVOF & Multi-scale \\
\hline 1.0 & 1.05 \\
\hline
\end{tabular}

Table 4: Comparative normalized computational time of the pure CLSVOF and the multiscale algorithm.

\begin{tabular}{r|ccc}
\hline & \multicolumn{3}{|c}{ Time [\%] } \\
\hline Step & Tag only & No Tag & All \\
\hline Interface tracking & 38.6 & 12.0 & 14.7 \\
\hline Momentum solve & 4.0 & 5.6 & 5.4 \\
\hline Pressure solve & 49.6 & 71.4 & 69.2 \\
\hline
\end{tabular}

Table 5: Relative computational time of different parts of the multi-scale algorithm.

normalized computational time with and without the multi-scale algorithm. The computational time is globally increased by $5 \%{ }^{4}$.

Table 5 shows more details about the repartition of the computational time is a single time-step. The tagging algorithm in particular is expected to be quite expensive, given the multiple mesh sweeps of the tag propagation. In consequence, the results are presented for the time-steps with droplet detection only, for time-steps without droplet detection and for all the timesteps regardless of the tagging call. These tests have been performed with a

\footnotetext{
${ }^{4}$ In several instances the computational time actually decreased in reason of a smaller number of pressure solver iterations: it is possible that the missing surface tension source terms may lead to a simpler matrix to inverse.
} 
fixed number of solver iterations, in order to focus on the effect of the multiscale added operations. It is clear that a time-step sees an increase from $12 \%$ to $38 \%$ of contribution from the multi-scale tagging algorithm. However, the average of the 10 time-steps leads to a relative $3 \%$ increase only. This results may also be affected by the refinement efficiency of the AMR. Globally, the computational cost of the methodology is very manageable.

In the sheet simulation of section 5.4, it is very likely that AMR would be entirely ineffective without the multi-scale algorithm. The number of effective points on the theoretical single-grid number ratio is roughly 0.10.2 , meaning that the global gain on computational resources leans heavily towards the multi-scale simulation. Moreover, the droplet tagging algorithm allow an on-the-run post-processing of the spray (seen in figure 21), while other approaches like the image post-processing seem to perform poorly (i.e. very high droplet rejection).

\section{Conclusion}

An innovative multi-scale methodology for the direct numerical simulation of atomization has been presented. It consists of three main items. The first is a coupled Level-Set/Volume-of-Fluid method (CLSVOF) for accurate capture of the primary atomization process. The second an adaptive mesh refinement technique (oct-tree AMR) to dynamically optimize the structured Cartesian mesh. The third consists of a particle tracking algorithm to capture droplet dynamics. An improved Eulerian-Lagrangian coupling has been developed to assure a smooth transition between the Eulerian and the Lagrangian modelling of the droplets, where both models approach their design limits. The overall procedure is tested on simplified numerical tests and validated on a planar liquid sheet assisted atomization case. The dedicated test cases show a good behaviour of the multi-scale methodology, in particular in the targeted middle-scales where small liquid inclusions are captured on few mesh cells. The simulation of the assisted atomization has been successfully performed, a comparison with the corresponding experience from ONERA has been possible. A very good qualitative agreement has been found: all the atomization processes, from large scale instabilities to small droplet dynamics have been reproduced by the DNS. A preliminary statistical spray analysis has been performed as well, showing a realistic and reasonably converged distribution of droplet sizes. 


\section{Acknowledgements}

The PARAMESH software used in this work was developed at the NASA Goddard Space Flight Center and Drexel University under NASA's HPCC and ESTO/CT projects and under grant NNG04GP79G from the NASA/AISR project.

The present study has been granted by the Foundation STAE-RTRAresearch program COFFECI. The authors are grateful to the STAE-RTRA Foundation for having funded this study and especially the post-doc of Dr B. DiPierro.

This work was granted access to the HPC resources of CINES under the allocation 2014-x20142b7264 made by GENCI.

The authors wish to thank the reviewers for their careful reading of the manuscript and their many insightful comments and suggestions which greatly improved the quality of the paper.

\section{References}

[1] B. Stapper, W. Sowa, G. Samuelsen, An experimental study of the effects of liquid properties on the breakup of a two-dimensional liquid sheet., Journal of Engineering for Gas Turbines and Power (114) (1992) 32-39.

[2] B. Stapper, G. Samuelsen, An experimental study of the breakup of a two dimensional liquid sheet in the presence of co-flow air shear., in: 28th Aerospace Sciences Meeting, 1990.

[3] A. Mansour, N. Chigier, Dynamic behavior of liquid sheets., Physics of Fluids A: Fluid Dynamics 3 (1991) 2971-2980.

[4] A. Mansour, N. Chigier, Disintegration of liquid sheets., Physics of Fluids A: Fluid Dynamics 2 (1990) 706-719.

[5] V. G. Fernandez, Experimental study of a liquid sheet disintegration in a high pressure environment, Ph.D. thesis, ISAE (2009).

[6] F. Jaegle, J.-M. Senoner, M. Garcia, F. Bismes, R. Lecourt, B. Cuenot, T. Poinsot, Eulerian and lagrangian spray simulations of an aeronautical multipoint injector, Proceedings of the Combustion Institute 33 (2) (2011) 2099-2107. doi:DOI:10.1016/j.proci.2010.07.027. 
[7] J. Senoner, M. Sanjose, T. Lederlin, F. Jaegle, M. Garcia, E. Riber, B. Cuenot, L. Gicquel, H. Pitsch, T. Poinsot, Eulerian and lagrangian large-eddy simulations of an evaporating two-phase flow, Comptes Rendus Mecanique 337 (6-7) (2009) 458-468.

[8] M. Sussman, E. Puckett, A coupled level set and volume of fluid method for computing 3d and axisymmetric incompressible two-phase flow., Journal of Computational Physics 162 (2000) 301-337.

[9] O. Desjardins, V. Moureau, E. Knudsen, M. Herrmann, H. Pitsch, Conservative level set/ghost fluid method for simulating primary atomization., in: 20th Annual Conference on Liquid Atomization and Spray Systems, in ILASS Americas, Vol. 238, 2007, pp. 8395-8416.

[10] K. Luo, C. Shao, Y. Yang, J. Fan, A mass conserving level set method for detailed numerical simulation of liquid atomization, Journal of Computational Physics 298 (0) (2015) 495 - 519. doi:http://dx.doi.org/10.1016/j.jcp.2015.06.009. URL http://www.sciencedirect.com/science/article/pii/ S0021999115003952

[11] R. Lebas, T. Menard, P. Beau, A. Berlemont, F. Demoulin, Numerical simulation of primary break-up and atomization: DNS and modelling study, International Journal of Multiphase Flow 35 (2009) 247-260.

[12] M. Gorokhovski, M. Herrmann, Modeling primary atomization, Annual Review of Fluid Mechanics 40 (2008) 343-366.

[13] O. Desjardin, V. Moureau, H. Pitsch, An accurate conservative level set/ghost fluid method for simulating turbulent atomization., Journal of Computational Physics 227 (18) (2008) 839-8416.

[14] M. Herrmann, A parallel eulerian interface tracking/lagrangian point particle multi-scale coupling procedure, Journal of Computational Physics 229 (2010) 745-759.

[15] G. Tomar, D. Fuster, S. Zaleski, S. Popinet, Multiscale simulations of primary atomization using gerris, Computers and Fluids 39 (10) (2010) 1864-1874.

URL http://gfs.sf .net/papers/tomar2010.pdf 
[16] M. Herrmann, A balanced force refinement level sel grid method for twophase flows on unstructured flow solver grid, Journal of Computational Physics 227 (2008) 2674-2706.

[17] D. Fuster, A. Bague, T. Boeck, L. LeMoyne, A. Leboissetier, S. Popinet, P. Ray, R. Scardovelli, S. Zaleski, Simulation of primary atomization with an octree adaptive mesh refinement and VOF method, International Journal of Multiphase Flow 35 (2009) 550-565.

[18] M. Sussman, A. S. Almgren, J. B. Bell, P. Colella, L. H. Howell, M. L. Welcome, An adaptive level set approach for incompressible two-phase flows, Journal of Computational Physics 148 (1999) 81-124.

[19] M. Sussman, A parallelized, adaptive algorithm for multiphase flows in general geometries, Computers and Structures 83 (6-7) (2005) 435-444.

[20] S. Popinet, S. Zaleski, Gerris: a tree- based adaptive solver for the incompressible euler equations in complex geometries., Journal of Computational Physics 190 (2) (2003) 572-600.

[21] S. Popinet, An accurate adaptive solver for surface tension driven interfacial flows., Journal of Computational Physics 228 (2009) 5838-5866.

[22] G. Pau, J. Bell, A. Almgren, K. Fagnan, M. Lijewski, An adaptive mesh refinement algorithm for compressible two-phase flow in porous media, Computational Geosciences 16 (3) (2012) 577-592. doi:10. 1007/s10596-011-9270-2. URL http://dx.doi.org/10.1007/s10596-011-9270-2

[23] D. Zuzio, J.-L. Estivalezes, An efficient block parallel amr method for two phase flow simulations, Computer and Fluids 44 (2011) 339-357.

[24] M. R. Maxey, J. J. Riley, Equation of motion for a small rigid sphere in a nonuniform flow, Physics of Fluids 26 (4) (1983) 883-889. doi:http://dx.doi.org/10.1063/1.864230. URL http://scitation.aip.org/content/aip/journal/pof1/26/ 4/10.1063/1.864230

[25] M. Pai, S. Subramaniam, A comprehensive probability density function formalism for multiphase flows, Journal of Fluid Mechanics 628 (2009) 181-228. doi:doi:10.1017/S002211200900617. 
[26] J. Capecelatro, O. Desjardins, An euler-lagrange strategy for simulating particle-laden flows, Journal of Computational Physics 238 (2013) 1 31. doi:http://dx.doi.org/10.1016/j.jcp.2012.12.015.

URL http://www.sciencedirect.com/science/article/pii/ S0021999112007462

[27] J.-M. Senoner, Large-eddy simulation of the two-phase flow in an aeronautical combustor using an euler-lagrange approach., Ph.D. thesis, Institut National Polytechnique de Toulouse, CERFACS (2010).

[28] R. H. Rangel, W. A. Sirignano, An evaluation of the point-source approximation in spray calculations., Numerical Heat Transfer, Part A: Applications 16 (1) (1989) 37-57. doi:10.1080/10407788908944705. URL http://dx.doi.org/10.1080/10407788908944705

[29] M. Herrmann, Detailed numerical simulations of the primary atomization of a turbulent liquid jet in crossflow, Journal of Engineering for Gas Turbines and Power 132 (6) (2010) 451-466. doi:10.1115/1.4000148. URL http://link. aip.org/link/?GTP/132/061506/1

[30] X. Li, M. Arienti, M. Soteriou, M. Sussman, Towards an efficient, highfidelity methodology for liquid jet atomization computations, AIAA, 2010-210 48th AIAA Aerospace Sciences Meeting 2010.

[31] M. Arienti, X. Li, C. Soteriou, C. Eckett, M. Sussman, R. Jensen, Coupled level-set/volume-of-fluid method for simualtaion of injector atomization, Journal of propulsion and power 29 (2013) 147-157.

[32] S. Apte, M. Gorokhovski, P. Moin, Les of atomizing spray with stochastic modeling of secondary break-up, International Journal of Multiphase Flow 29 (2003) 1503-1522.

[33] M. A. Gorokhovski, V. L. Saveliev, Analyses of kolmogorov's model of breakup and its application into lagrangian computation of liquid sprays under air-blast atomization, J. Physics of Fluids 15 (1) (2003) 184-192. doi:DOI: $10.1063 / 1.1527914$.

URL http://dx.doi.org/doi/10.1063/1.1527914

[34] T. Menard, S. Tanguy, A. Berlemont, Coupling Level set/VOF/Ghost fluid methods: Validation and application to 3d simulation of the pri- 
mary break-up of a liquid jet., International Journal of Multiphase Flow 33 (5) (2007) 510-524.

[35] P. MacNeice, K. M. Olson, C. Mobarry, R. de Fainchtein, C. Packer, Paramesh: A parallel adaptive mesh refinement community toolkit, Computer Physics Communications 126 (3) (2000) 330 - 354. doi:http://dx.doi.org/10.1016/S0010-4655(99)00501-9. URL http://www.sciencedirect.com/science/article/pii/ S0010465599005019

[36] A. Sarthou, D. Zuzio, J.-L. Estivalezes, Multiscale euler-lagrange method for parallel simulation of atomization induced by air-blast planar injectors, 21st AIAA Computational fluid Dynamics Conference, 2013.

[37] J. López, J. Hernàndez, Analytical and geometrical tools for $3 \mathrm{~d}$ volume of fluid methods in general grids, Journal of Computational Physics 227 (12) (2008) 5939 - 5948. doi:http: //dx.doi.org/10.1016/j.jcp.2008.03.010. URL http://www.sciencedirect.com/science/article/pii/ S0021999108001629

[38] D. S. Balsara, Divergence-free adaptive mesh refinement for magnetohydrodynamics., Journal of Computational Physics 174 (2) (2001) 614648.

[39] K. M. Olson, P. MacNeice, An overview of the paramesh amr software package and some of its applications (2005) 315-330doi:10.1007/ 3-540-27039-6_22.

URL http://dx.doi .org/10.1007/3-540-27039-6_22

[40] M. Maxey, B. Patel, Localized force representations for particles sedimenting in stokes flow, International Journal of Multiphase Flow 27 (9) (2001) 1603-1626. doi:http://dx.doi.org/10.1016/ S0301-9322(01)00014-3.

URL http://wWW.sciencedirect.com/science/article/pii/ S0301932201000143

[41] Y. Ling, S. Zaleski, R. Scardovelli, Multiscale simulation of atomization with small droplets represented by a lagrangian point-particle model, International Journal of Multiphase Flow 76 (2015) 122 - 143. doi: 
http://dx.doi.org/10.1016/j.ijmultiphaseflow.2015.07.002.

URL http://wwW.sciencedirect.com/science/article/pii/ S0301932215001524

[42] L. Schiller, A. Nauman, A drag coefficient correlation, VDI Zeitung 77 (1935) 318-320.

[43] R. D. Felice, A relationship for the wall effect on the settling velocity of a sphere at any flow regime, International Journal of Multiphase Flow 22 (3) (1996) 527 - 533. doi:http: //dx.doi.org/10.1016/0301-9322(96)00004-3.

URL http://wWw.sciencedirect.com/science/article/pii/ 0301932296000043

[44] B. Déjean, P. Berthoumieu, P. Gajan, Experimental study on the influence of the liquid and air thicknesses on a planar air-blasted liquid sheet, in: 25th European Conference - Liquid Atomization \& Spray Systems ILASS 2013, CHANIA, Greece, 2013. URL https://hal-onera. archives-ouvertes.fr/hal-01058399

[45] A. Lozano, F. Barreras, G. Hauke, C. Dopazo, Longitudinal instabilities in a air-blasted liquid sheet., Journal of Fluid Mechanics 437 (2001) $143-173$.

[46] F. Couderc, Développement d'un code de calcul pour la simulation d'écoulements de fluides non miscibles. application à la désintégration assistée d'un jet liquide par un courant gazeux., Ph.D. thesis, ENSAE (2007).

[47] G. Blanchard, Modlisation et simulation multichelle de l'atomisation d'une nappe liquide cisaille., Ph.D. thesis, ISAE (2014).

[48] D. Zuzio, J.-L. Estivalezes, A parallel adaptive projection method for incompressible two phase flows, in: ICCFD 2010 Refereed Conference Proceedings, ISSN: 2070-3740 and ISSN: 2070-3724, 2010. 